\title{
ECRad: An electron cyclotron radiation transport solver for advanced data analysis in
} thermal and non-thermal fusion plasmas

Denk, S. S.; Fischer, R.; Poli, E.; Maj, O.; Nielsen, S. K.; Rasmussen, J.; Stejner, M.; Willensdorfer, M.

Published in:

Computer Physics Communications

Link to article, DOI:

10.1016/j.cpc.2020.107175

Publication date:

2020

Document Version

Peer reviewed version

Link back to DTU Orbit

Citation (APA):

Denk, S. S., Fischer, R., Poli, E., Maj, O., Nielsen, S. K., Rasmussen, J., Stejner, M., \& Willensdorfer, M. (2020). ECRad: An electron cyclotron radiation transport solver for advanced data analysis in thermal and non-thermal fusion plasmas. Computer Physics Communications, 253, [107175]. https://doi.org/10.1016/j.cpc.2020.107175

\section{General rights}

Copyright and moral rights for the publications made accessible in the public portal are retained by the authors and/or other copyright owners and it is a condition of accessing publications that users recognise and abide by the legal requirements associated with these rights.

- Users may download and print one copy of any publication from the public portal for the purpose of private study or research.

- You may not further distribute the material or use it for any profit-making activity or commercial gain

- You may freely distribute the URL identifying the publication in the public portal 


\section{Journal Pre-proof}

ECRad: An electron cyclotron radiation transport solver for advanced data analysis in thermal and non-thermal fusion plasmas

S.S. Denk, R. Fischer, E. Poli, O. Maj, S.K. Nielsen, J. Rasmussen,

M. Stejner, M. Willensdorfer, the ASDEX Upgrade Team

PII: $\quad$ S0010-4655(20)30029-1

DOI: $\quad$ https://doi.org/10.1016/j.cpc.2020.107175

Reference: COMPHY 107175

To appear in: Computer Physics Communications

Received date : 18 July 2019

Revised date: 6 December 2019

Accepted date: 14 January 2020

Please cite this article as: S.S. Denk, R. Fischer, E. Poli et al., ECRad: An electron cyclotron radiation transport solver for advanced data analysis in thermal and non-thermal fusion plasmas, Computer Physics Communications (2020), doi: https://doi.org/10.1016/j.cpc.2020.107175.

This is a PDF file of an article that has undergone enhancements after acceptance, such as the addition of a cover page and metadata, and formatting for readability, but it is not yet the definitive version of record. This version will undergo additional copyediting, typesetting and review before it is published in its final form, but we are providing this version to give early visibility of the article. Please note that, during the production process, errors may be discovered which could affect the content, and all legal disclaimers that apply to the journal pertain.

(C) 2020 Elsevier B.V. All rights reserved. 
1

2

3

4

5

6

7

8

9

10

11

12

13

14

15

16

17

18

19

20

21

22

23

24

25

26

27

28

29

30

31

32

33

34

35

36

37

38

39

40

41

42

43

44

45

46

47

48

49

50

51

52

53

54

\title{
ECRad: An Electron Cyclotron Radiation transport solver for Advanced Data analysis in thermal and non-thermal fusion plasmas
}

S. S. Denk ${ }^{\mathrm{a}, *}$, R. Fischer ${ }^{\mathrm{a}}$, E. Poli ${ }^{\mathrm{a}}$, O. Maj ${ }^{\mathrm{a}}$, S. K. Nielsen ${ }^{\mathrm{b}}$, J. Rasmussen $^{\mathrm{b}}$, M. Stejner ${ }^{b}$, M. Willensdorfer ${ }^{a}$, the ASDEX Upgrade Team ${ }^{1}$

${ }^{a}$ Max Planck Institute for Plasma Physics, Boltzmannstr. 2, 85748 Garching, Germany

${ }^{b}$ Technical University of Denmark, Department of Physics, Kgs. Lyngby, Denmark

\begin{abstract}
\end{abstract}
We present a code for the large-scale data analysis of electron cyclotron emission (ECE) measurements from magnetized plasmas called ECRad - the Electron Cyclotron Radiation transport model for Advanced Data analysis. Its key features are low computational cost, high robustness and the capability to predict ECE spectra of plasmas with non-thermal electron populations. This is accomplished by combining the absorption coefficient given by F. Albajar et al., PPCF 49, 15 (2007) and a corresponding emissivity for the radiation transport with geometrical optics ray tracing for the computation of the diagnostic line of sight. Another important aspect of ECRad is that it has passed a large amount of verification tests against real measurements by conventional, oblique and ECE imaging diagnostics.

This paper explains the physical model of ECRad and its implementation. Furthermore, it is discussed how the code can be used for the inference of the electron temperature from ECE measurements and how an oblique ECE diagnostic can be cross-calibrated with ECRad.

Keywords: Electron Cyclotron Emission, Radiation Transport, Data Analysis

\section{Introduction}

Electron Cyclotron Emission (ECE) is one of the few diagnostic techniques capable of delivering spatially and temporarily highly resolved measurements of the electron temperature $\left(T_{\mathrm{e}}\right)$ of magnetized fusion plasmas. Accordingly, an ECE diagnostic can be found at nearly every major magnetic confined fusion device, e.g. JET $[1,2,3]$, ASDEX Upgrade [4, 5, 6], DIII-D [7, 8, 9], W7-X [10], LHD $[11,12]$ and KSTAR $[13,14]$. Conventional ECE diagnostics are primarily used to measure the $T_{\mathrm{e}}$ profile and large $T_{\mathrm{e}}$ fluctuations. Additionally, the ECE

* Corresponding author

${ }^{1}$ See author list of "MEYER, H. et al, Nucl. Fusion accepted (2019)

Preprint submitted to Elsevier

December 6, 2019 
technique is employed to measure the poloidal and toroidal motion and spectra of magnetohydrodynamic (MHD) modes by ECE imaging diagnostics [15, 8, 14]. Turbulent $T_{\mathrm{e}}$ fluctuations are resolved with correlation ECE [16].

The measurements of these diagnostics can be interpreted by identifying the observed radiation temperature with $T_{\mathrm{e}}$ at the cold resonance position. This is based on the application of the black-body radiation law at the radial position, where the observed frequency matches the electron cyclotron frequency or one of its harmonics. However, this approach can become inaccurate if, for example, one is interested in the $T_{\mathrm{e}}$ profile near the plasma edge, where the plasma is optically thin [4]. Another situation, where this approach is inapplicable, occurs if the line of sight (LOS) of the diagnostic is not perpendicular to the magnetic field. In this case the LOS of the diagnostic can be strongly refracted and the emission is affected by the Doppler-shift $[17,18]$. ECE has also been used to study non-thermal electron distribution functions. Here various diagnostic setups have been realized, like a high-field side (HFS) ECE at TCV [19], an oblique ECE at JET [3] and, recently, vertical ECE systems at TCV [20] and COMPASS [21]. In all these cases and especially for the quantitative interpretation of non-thermal ECE measurements [22], an accurate model of the radiation transport [23] in the plasma is mandatory.

Several radiation transport codes for ECE are available, e.g. SPECE[24], TRAVIS[25] and NOTEC[26]. These codes address mainly diagnostic design $[27]$ and the in-depth analysis of single ECE spectra $[28,29,30]$. In this paper we present a radiation transport solver specifically designed for the large-scale analysis of ECE spectra of toroidally symmetric devices: The Electron Cyclotron Radiation transport model for advanced data analysis (ECRad). At the time of this writing, five years have passed since the code was first deployed for the routine analysis of the ECE measurements within the ASDEX Upgrade Integrated Data Analysis (IDA) framework [31]. It analyzes, among the data of other diagnostics, the measurements of the profile radiometer with a standard resolution of one millisecond for the majority of discharges performed at ASDEX Upgrade [4, 32]. During these five years the code has undergone continuous development to be capable of describing the majority of ECE spectra recorded by the various diagnostics available at ASDEX Upgrade. Apart from the analysis of the profile radiometer, the code also assisted the in-depth analysis of ECE imaging measurements $[18,33,34,35]$, the interpretation of correlation ECE measurements [6] and the design of a recently deployed correlation ECE setup at ASDEX Upgrade [36].

While the physical model of the code is resembling the main three alternative codes mentioned above, the key feature of ECRad is the efficient implementation and its optimization towards the everyday ECE analysis. ECRad is not restricted to ECE spectra of thermal plasmas. The code is capable of computing ECE spectra for bounce-averaged electron momentum distribution function profiles. This has been used extensively in Ref. [37] to benchmark Fokker-Planck models for electron cyclotron current drive against measurements of both radial and oblique ECE diagnostics.

The goal of the present paper is to describe the details of the physical model 
of ECRad and the key aspects of the implementation which makes the largescale analysis of ECE measurements computationally feasible. The paper is structured into four parts. In section 2 the underlying physical model of ECRad is presented. In Section 3 the implementation details are explained. Section 4 discusses several applications of ECRad. Conclusions are drawn in section 5.

\section{Theory}

ECRad performs two main tasks: Solving the geometrical optics equations and the radiation transport equation. In the following the details of these two steps are explained.

\subsection{Wave propagation}

In the geometrical optics limit the trajectory of an electromagnetic wave in the electron cyclotron range of frequencies is governed by the following Hamiltonian system[38]. Here we follow the notation of Ref. [39], but drop the second order terms that describe the diffraction of the waves:

$$
\frac{\mathrm{d} \boldsymbol{x}}{\mathrm{d} s}=\left.\frac{\partial \Lambda / \partial \boldsymbol{N}}{|\partial \Lambda / \partial \boldsymbol{N}|}\right|_{\Lambda=0} ; \quad \frac{\mathrm{d} \boldsymbol{N}}{\mathrm{d} s}=-\left.\frac{\partial \Lambda / \partial \boldsymbol{x}}{|\partial \Lambda / \partial \boldsymbol{N}|}\right|_{\Lambda=0} .
$$

In the previous equation $s$ is the arc length of the ray and $\boldsymbol{x}$ is the spatial coordinate. The second canonical coordinate is $\boldsymbol{N} \equiv \frac{c_{0} \boldsymbol{k}}{\omega}$ with $\boldsymbol{k}$ the wave vector and $\Lambda$ represents the cold plasma dispersion relation give by equation (2).

The index of refraction $N_{\mathrm{s}, \omega}$ derived from the dispersion relation depends on the angular frequency of the waves $\omega$, electron density $\left(n_{\mathrm{e}}\right)$ and, in case of $\mathrm{X}$-mode polarization, also on the magnetic field strength $B=|\boldsymbol{B}|$. The cold dispersion relation $\Lambda(\boldsymbol{x}, \boldsymbol{k}, \omega)$ has two roots, which correspond to the extraordinary mode (X-mode) and the ordinary mode (O-mode):

$$
\Lambda(\boldsymbol{x}, \boldsymbol{N}, \omega)=|\boldsymbol{N}|^{2}-N_{\mathrm{s}, \omega}^{2}\left(X, Y, N_{\|}\right)
$$

with $N_{\mathrm{s}, \omega}$ the cold plasma refractive index [40]

$$
N_{\mathrm{s}, \omega}\left(X, Y, N_{\|}\right)=1-X-X Y^{2} \frac{1+N_{\|}^{2} \pm \Delta}{2\left(1-X-Y^{2}\right)},
$$

where

$$
\Delta \equiv \sqrt{\left(1-N_{\|}^{2}\right)^{2}+4 N_{\|}^{2} \frac{1-X}{Y^{2}}}
$$

In equation (2) the normalized plasma frequency $X \equiv \frac{\omega_{\mathrm{p}}^{2}}{\omega^{2}}$ with plasma frequency $\omega_{\mathrm{p}}=\sqrt{\frac{e^{2} n_{\mathrm{e}}}{\epsilon_{\mathrm{e}} m_{\mathrm{e}, 0}}}$ and the normalized cyclotron frequency $Y \equiv \frac{\omega_{c, 0}}{\omega}$ and the cyclotron frequency $\omega_{\mathrm{c}, 0}=\frac{e B}{m_{\mathrm{e}, 0}}$ were introduced. The vacuum permittivity $\epsilon_{0}$ 
has its usual notation. The angle between the wave vector and the magnetic field is

$$
\theta=\arccos \left(\frac{\boldsymbol{k} \cdot \boldsymbol{B}}{|\boldsymbol{k}| \cdot|\boldsymbol{B}|}\right)
$$

The component of $\boldsymbol{N}$ parallel to the magnetic field is denoted as $N_{\|} \equiv|N| \cos \theta=$ $\frac{\boldsymbol{N} \cdot \boldsymbol{B}}{|\boldsymbol{B}|}$ and $N_{\perp} \equiv|\boldsymbol{N} \sin \theta|$ is defined correspondingly. The " + " in equation (3) corresponds to X-mode and "-" to O-mode.

The two relations in equation (1) form a system of two coupled, ordinary, three-dimensional differential equations, which have to be solved numerically. The ray paths given by the Hamiltonian system fulfill reciprocity. This is important for modeling ECE diagnostics where the origin of the ECE is not known, while the position of the antenna is. Due to reciprocity of the Hamiltonian system, the rays can be launched from the ECE antenna to determine the path of the radiation traveling through the plasma to the ECE antenna.

It is common practice to disregard kinetic effects in the computation of the trajectory of cyclotron waves with ray and beam tracing codes [41, 42, 39, 43, 44]. There is, however, an important exception where this practice is inaccurate. Near a cut-off layer, at which the refractive index becomes purely imaginary, there can be a considerable influence by kinetic effects [45]. Due to the large speed of the electrons, relativistic effects allow propagation of electron cyclotron waves even if the refractive index given by the cold dispersion relation is purely imaginary. Solving this problem rigorously bears two problems. First, the group velocity of the wave approaches zero [46]. Second, there is no analytical solution to the fully relativistic dispersion relation and an extensive numerical approach is required. These problems can be overcome by introducing approximations, e.g. Ref. [25], but the numerical costs are still substantial. Including finite temperature effects in ray tracing for the large-scale data analysis of ECE measurements is not feasible at the present time. To consider finite temperature effects near cut-offs, a less rigorous approach is employed in ECRad. This is accomplished by introducing a weakly relativistic correction to the electron mass in both, the cyclotron and the plasma frequency [45]

$$
\tilde{m}_{\mathrm{e}} \equiv m_{\mathrm{e}, 0} \sqrt{1+\frac{5}{\mu}}, \quad \mu=\frac{m_{\mathrm{e}, 0} c_{0}^{2}}{k_{\mathrm{b}} T_{\mathrm{e}}}, \quad \tilde{\omega}_{\mathrm{p}}=\sqrt{\frac{n_{\mathrm{e}} e^{2}}{\epsilon_{0} \tilde{m}_{\mathrm{e}}}}, \quad \tilde{\omega}_{\mathrm{c}}=\frac{e B}{\tilde{m}_{\mathrm{e}}} .
$$

The Boltzmann constant has its usual notation $k_{\mathrm{b}}$ in equation $(6)$.

The same approach is already implemented in TORBEAM [42], where it is used for the determination of the cut-off layer for reflectometry. In close proximity of cut-off layers this treatment is exact [47], but the approximation breaks down far away from cut-off. To demonstrate that the correction given by equation (6) is meaningful and generally leads to an improvement of the ray paths an example will be discussed in the following.

As the scenario we choose the ASDEX Upgrade discharge \#35322 at $t=$ $2.168 \mathrm{~s}$, in which the on axis magnetic field strength is $2.5 \mathrm{~T}$. For the line of 


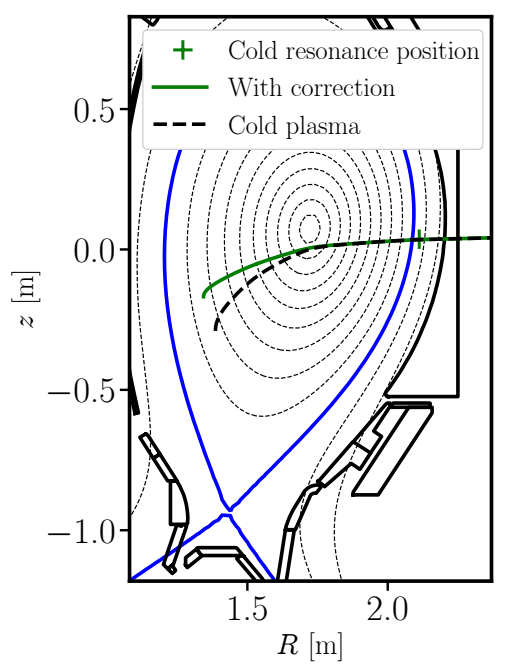

Figure 1: The figure shows the poloidal cross section of ASDEX Upgrade and two LOS of an ECE channel with $\rho_{\text {pol,res }}=1.07$ for discharge \#35322 and $t=2.168 \mathrm{~s}$. The green line depicts the LOS computed with the correction given by equation (6) and the black dashed line corresponds to the LOS that is obtained if the correction is omitted. The figure also shows the contours of $\rho_{\text {pol }}$ and the ASDEX Upgrade vessel wall. The separatrix is indicated in blue.

sight geometry and measurement frequency a channel of the ASDEX Upgrade profile radiometer with $f=105 \mathrm{GHz}$ is selected. This frequency corresponds to a cold resonance position to the second harmonic in term of the square root of the normalized flux $\rho_{\text {pol,res }}=1.07$. This scenario is particularly interesting, because a comparatively large $T_{\mathrm{e}}$ at the plasma center of $8.5 \mathrm{keV}$ causes warm plasma effects to be relevant for ray tracing. Figure 1 shows the ray trajectories computed by ECRad with (green solid) and without (black dashed) the correction given by equation (6). There is a clear deviation between the two rays. To demonstrate that the approximation actually provides better results, figure 2 compares the cold plasma and the corrected refractive index to the real part of the refractive index determined rigorously from the warm dielectric tensor as described in Ref. [48]. The refractive index corrected according to equation (6) is much closer to the real part of the warm refractive index than the cold one.

This finding is not limited to the scenario discussed here, but is generally true with one exception. Near a resonance the real part of the fully relativistic refractive index tends to be lower than the one with the correction and it is then closer to the cold refractive index. Given the results from past studies $[49,40,46]$, it is, however, not expected that the effect of the resonance on 


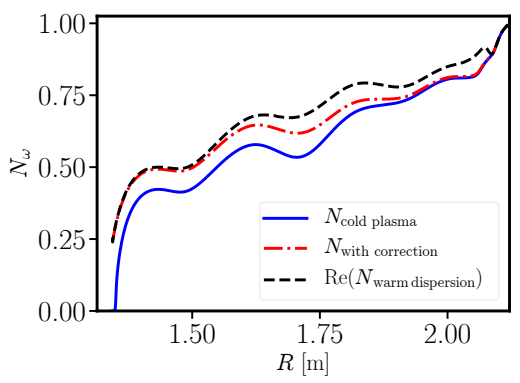

Figure 2: Comparison between the Appleton-Hartree cold plasma refractive index $N_{\text {cold plasma, }}$, the refractive index $N_{\text {with correction, where the correction given by equation (6) }}$ is considered, and the real part of the refractive index derived from the fully relativistic dispersion relation $\operatorname{Re}\left(N_{\text {warm dispersion }}\right)$. All three refractive indices are given as functions of the major radius.

the wave propagation is significant for the large scale data analysis of ECE measurements. An exception is the scenario, where resonance reduces the real part of refractive index to zero, but such fringe cases are anyways beyond the scope of ECRad.

\subsection{The radiation transport equation for electron cyclotron waves}

The radiation transport equation is given by Ref. [23]:

$$
\frac{\mathrm{d}}{\mathrm{d} s} \frac{I_{\omega}(s)}{N_{\omega, \text { ray }}^{2}(s)}=\frac{1}{N_{\omega, \text { ray }}^{2}(s)}\left(j_{\omega}(s)-\alpha_{\omega}(s) I_{\omega}(s)\right) .
$$

The coordinate of the path along which the radiation propagates is the arclength $s$ and $N_{\omega, \text { ray }}$ is the ray refractive index [23]. The absorption coefficient is denoted as $\alpha_{\omega}(s)$ and captures the relative loss of intensity due to wave absorption. The source term is the emissivity $j_{\omega}(s)$. Section 2.3 shows how these two quantities are calculated. Integrating equation (7) over the path of the ray yields the intensity $I_{\omega}\left(s_{\text {ow }}\right)$ for a measured frequency $\omega$ at the position of the outer wall $s_{\mathrm{ow}}$, where the radiation leaves the plasma vessel and is detected.

For the interpretation of ECE measurements the values $I_{\omega}$ inside the plasma are irrelevant and only $I_{\omega}\left(s_{\text {ow }}\right)$ is of importance. Since $s_{\text {ow }}$ is in vacuum, $N_{\omega, \text { ray }}$ can be set to one in equation (7). This can be proven by inserting Kirchhoff's law (cf. equation (14) below) into equation (7):

$$
\frac{\mathrm{d}}{\mathrm{d} s} \frac{I_{\omega}(s)}{N_{\omega, \text { ray }}^{2}(s)}=\alpha_{\omega}(s) I_{\mathrm{BB}, \omega}-\alpha_{\omega}(s) \frac{I_{\omega}(s)}{N_{\omega, \text { ray }}^{2}(s)},
$$

where $I_{\mathrm{BB}, \omega}$ denotes the black body intensity. Introducing $\tilde{I}_{\omega} \equiv I_{\omega} / N_{\omega, \text { ray }}^{2}$ renders equation (8) independent of $N_{\omega, \text { ray }}$. In vacuum at $s=s_{\text {ow }}$ the ray refractive index is one and, therefore, $I_{\omega}\left(s_{\mathrm{OW}}\right)=\tilde{I}_{\omega}\left(s_{\mathrm{ow}}\right)$. Although Kirchhoff's 
law was considered in this derivation, it can be shown that this approximation also holds for non-thermal distributions (cf. equation (9)).

\subsection{Electron cyclotron emission and absorption}

To solve the radiation transport equation (7) the emissivity $j_{\omega}(s)$ and the absorption coefficient $\alpha_{\omega}(s)$ have to be calculated. In a tenuous plasma, where dispersion is negligible, the collective motion of the electrons can be neglected and the emissivity and absorption coefficient can be calculated as the sum of the contributions of each electron. Hence, the emissivity is given by the velocity space integral of the single electron emissivity weighted with the distribution function [50]. This approach requires that refractive index $N_{\mathrm{s}, \omega} \approx 1$. For plasma scenarios typical of ASDEX Upgrade and for the frequency range for which ECE is a useful diagnostic tool this criterion does usually not apply. Hence, the dielectric properties of the plasma have to be retained when calculating the emissivity. This requires the knowledge of the complex wave vector $\boldsymbol{k}$, which fulfills the fully relativistic dispersion relation locally. However, to obtain the complex wave vector it is necessary to solve the fully relativistic dispersion relation for the complex refractive index [48]. To date no analytical solution to this problem is available and it has to be solved numerically.

Unlike the cold plasma dispersion relation, the fully relativistic dispersion relation exhibits more than two roots. Aside from roots that correspond to the $\mathrm{X}$ - and $\mathrm{O}$-mode there are also roots that belong to electrostatic Bernstein waves [40]. Especially close to a resonance it is possible that the dispersion relation has two or more roots that lie close together [51]. At the present time there is no scheme readily available that can reliably identify the root for which the absorption coefficient is currently needed. Although a comparatively robust solution to this problem exists [48] the success rate of this method proved insufficient in practice for the large-scale data analysis targeted by ECRad. Hence, a compromise between the complete treatment and the tenuous plasma approach is needed.

A viable approximation for the absorption coefficient that retains the dielectric properties of the cold plasma is proposed in Ref. [52]. This approach can inherently only consider the electromagnetic energy flux of the wave, while the so-called sloshing flux, which is non-zero only if finite temperature effects are included in the dispersion relation, is neglected [52]. Furthermore, the refractive index $N_{\mathrm{s}, \omega}$ of the plasma and the polarization of the wave only account for the cold dielectric tensor and the correction of the electron mass introduced in equation (6). The advantage is that the absorption coefficient and the emissivity can be expressed as an integral in momentum space that can be easily and robustly solved numerically. With these approximations it is possible to derive the emissivity of the $n$-th harmonic from the absorption coefficient given in Ref. [52] and from equation (2.9) of Ref. [38]. The full derivation can be found in 
appendix Appendix A:

$$
\begin{gathered}
j_{\omega, n}(s)=\frac{N_{\omega, \mathrm{ray}}^{2} \omega_{\mathrm{p}, 0}^{2} \omega}{4 \pi c_{0}^{3}} \iint\left(\frac{n}{\bar{\omega} N_{\perp}}\right)^{2}\left|\left(\tilde{e}_{x}+\frac{\bar{\omega} N_{\perp}}{n} u_{\|} \tilde{e}_{z}\right) J_{n}(b)-\frac{i b}{n} J_{n}^{\prime}(b) \tilde{e}_{y}\right|^{2} \times \\
f\left(s, u_{\perp}, u_{\|}\right) \delta\left(\gamma-u_{\|} N_{\|}-\frac{n}{\bar{\omega}}\right) \frac{u_{\perp}}{\gamma} \mathrm{d} u_{\perp} \mathrm{d} u_{\|} .
\end{gathered}
$$

The absorption coefficient for harmonic number $n$ can directly be adopted from Ref. [52]:

$$
\begin{gathered}
\alpha_{\omega, n}(s)=-2 \pi^{2} \frac{\omega_{\mathrm{p}, 0}^{2}}{c_{0} \omega} \iint\left(\frac{n}{\bar{\omega} N_{\perp}}\right)^{2}\left|\left(\tilde{e}_{x}+\frac{\bar{\omega} N_{\perp}}{n} u_{\|} \tilde{e}_{z}\right) J_{n}(b)-\frac{i b}{n} J_{n}^{\prime}(b) \tilde{e}_{y}\right|^{2} \times \\
\hat{R} f\left(s, u_{\perp}, u_{\|}\right) \delta\left(\gamma-u_{\|} N_{\|}-\frac{n}{\bar{\omega}}\right) \frac{u_{\perp}}{\gamma} \mathrm{d} u_{\perp} \mathrm{d} u_{\|} .
\end{gathered}
$$

Both, the emissivity and the absorption coefficient contain an integral in cylindrical, dimensionless momentum space, with $u_{\perp / \|}=p_{\perp / \|} /\left(c_{0} m_{\mathrm{e}, 0}\right)$ the (dimensionless) momentum perpendicular/ parallel to the magnetic field. The Lorentz factor is denoted as $\gamma=\sqrt{1+u_{\perp}^{2}+u_{\|}^{2}}$ and $\bar{\omega} \equiv \frac{\omega}{\omega_{c, 0}}$. The distribution function $f$ can be an arbitrary, gyrotropic distribution. The coordinate system for the wave electric field $\boldsymbol{E}$ normalized with the Poynting flux $\boldsymbol{S}$

$$
\tilde{\boldsymbol{e}}=\left(\tilde{e}_{x}, \tilde{e}_{y}, \tilde{e}_{z}\right)=\frac{\boldsymbol{E}}{\sqrt{4 \pi|\boldsymbol{S}| / c}}
$$

follows the convention used by Stix, where the $x$ and $z$ axes are chosen such that $\boldsymbol{k}$ lies in the $x-z$ plane and the $z$ axis is aligned with $\boldsymbol{B}$ [53]. The Bessel function of first kind is denoted as $J_{n}(b)$ with $n$-th order and $b \equiv \bar{\omega} N_{\perp} u_{\perp}$. Note that most of these quantities depend implicitly on the ray coordinate $s$ and that the ray refractive index $N_{\omega, \text { ray }}$ can be set to one as described in the previous section. Furthermore, it should be noted that all quantities derived from the cold dispersion relation, i.e. $N_{\perp}, N_{\|}, N_{\omega}$ and $\tilde{e}$, incorporate the weakly relativistic correction of the electron rest mass, introduced in equation (6).

The absorption coefficient distinguishes itself from the emissivity by a factor and the operator $\hat{R}$ that is applied onto the electron distribution function.

$$
\hat{R} \equiv \frac{n}{\bar{\omega}} \frac{1}{u_{\perp}} \frac{\partial}{\partial u_{\perp}}+N_{\|} \frac{\partial}{\partial u_{\|}} .
$$

The distribution function can be arbitrarily chosen in equations (9) and (10).

To obtain the absorption coefficient $\alpha_{\omega}$ and the emissivity $j_{\omega}$ it is necessary to sum up the contributions of the individual harmonics

$$
\alpha_{\omega}=\sum_{n=1}^{\infty} \alpha_{\omega, n} ; \quad j_{\omega}=\sum_{n=1}^{\infty} j_{\omega, n} .
$$


For the majority of the ASDEX Upgrade discharges the electrons can be approximated to be thermally distributed, which allows the absorption coefficient to be derived from the emissivity via Kirchhoff's law [40] and vice versa:

$$
\alpha_{\omega}(s)=\frac{j_{\omega, n}(s)}{I_{\mathrm{BB}, \omega}(s) N_{\omega, \text { ray }}^{2}} \approx j_{\omega, n}(s) \frac{8 \pi^{3} c_{0}^{2}}{\omega^{2} k_{\mathrm{b}} T_{\mathrm{e}} N_{\omega, \text { ray }}^{2}} .
$$

The Rayleigh-Jeans approximation [50] for $I_{\mathrm{BB}, \omega}$ yields the expression given on the right side of equation (14). The Boltzmann constant is denoted as $k_{\mathrm{b}}$.

The omission of the sloshing flux and the reliance on the cold plasma approximation for the refractive index and wave polarization restrict the validity of equations (9) and (10) to harmonics with $n>1$, and $n>2$ if $\omega_{\mathrm{c}} \geqslant \omega_{\mathrm{p}}$ $[54,52]$. Since ECE measurements of the fundamental resonance $(n=1)$ are not common in most current magnetic confined fusion experiments, the inability to calculate the emissivity and absorption coefficient for the fundamental harmonic poses only a minor limitation at the present time. The second condition which applies for the second harmonic could pose a problem as $\omega_{\mathrm{c}} \approx \omega_{\mathrm{p}}$ for most plasmas. However, it was shown in Ref. [32] that the performance of equations (9) and (10) is adequate for the purposes of data analysis even if $\omega_{\mathrm{c}, 0} \approx \omega_{\mathrm{p}}$ and $n=2$. It should be noted that for second harmonic emission measurements in future devices like ITER the validity of these approximations for the emissivity and the absorption coefficient should be reexamined. For the interpretation of fundamental ECE measurements new expressions are needed.

\subsection{The birthplace distribution of observed intensity}

In classical ECE analysis each measurement frequency is mapped to its cold resonance position. Hence, there is a one-to-one correspondence between frequency and position. If broadening effects are to be considered in the analysis, this one-to-one relation is no longer applicable. Instead, for each measurement there is a distribution of measurement positions. Knowing this birthplace distribution of observed intensity $[17,18]$ can be very useful for understanding ECE measurements and it is provided as a standard output of ECRad. It is defined by

$$
D_{\omega}(s)=\frac{j_{\omega}(s) \mathcal{T}_{\omega}(s)}{I_{\omega}\left(s_{\mathrm{ow}}\right)}
$$

where $\mathcal{T}_{\omega}(s)$ is the transmittance of the plasma for waves at frequency $\omega$ along the ray path from point $s$ to the observation point $s_{\text {ow }}$ :

$$
\mathcal{T}_{\omega}(s)=\mathrm{e}^{-\tau_{\omega}(s)}=\exp \left(-\int_{s}^{s_{\mathrm{ow}}} \alpha_{\omega}\left(s^{\prime}\right) \mathrm{d} s^{\prime}\right)
$$

Functions similar to the birthplace distribution of observed intensity, although not normalized, have been used in various studies (see e.g. Ref. [55]) and numerous aliases like "total emissivity" [56], "actual emission" [4] or "emitting profile" [57] can be found in the literature. 


\section{Implementation}

ECRad is a Fortran90 code which utilizes OpenMP parallelization to solve the geometrical optics- and the radiation transport equation for several frequencies simultaneously. It was designed to be a tool for the everyday analysis of ECE measurements, including the inference of $T_{\mathrm{e}}$ from ECE measurements, cross-calibration of ECE diagnostics or the determination of the birthplace distribution. To fulfill this role the code has to be fast, robust and straightforward to use. For example in the IDA used at ASDEX Upgrade [31], it is not uncommon that every forward model has to be executed several thousands of times for every single time point. Simultaneously, the total computational time of IDA for each time point should not exceed more than a couple of minutes. Furthermore, to support gradient based optimization in the inference of $T_{\mathrm{e}}$ it is important that the radiation temperatures $\left(T_{\mathrm{rad}}\right)$ predicted by ECRad are continuously differentiable with respect to the input $T_{\mathrm{e}}$ profile. This requirement inhibits the usage of methods like root finding, adaptive step size algorithms and any technique reliant on random numbers. ECRad is a unique code, because it is able to meet all the requirements above.

\subsection{Main structure}

The workflow of the code has four main segments: Initialization, ray tracing, radiation transport and post-processing. In the initialization phase interpolating splines are computed for the input data and the antenna pattern is translated into the initial conditions for the ray tracing. The geometrical optics equations are solved in the ray tracing phase, in which also the grid for the radiation transport equation is prepared. It is then solved in the subsequent phase. The post-processing segment is optional. It provides the birthplace distributions and warm resonance positions.

If ECRad is used as stand-alone program through its Python [58] GUI, it will go through each of the four phases one after the other, where the postprocessing step can optionally be skipped. If ECRad is used as a library only the radiation transport equation can be solved in subsequent computations of $T_{\text {rad }}$, once initialization and ray tracing have been performed.

\subsection{Input}

ECRad requires four two-dimensional matrices describing a poloidal slice of the magnetic equilibrium $\left(B_{\mathrm{r}}, B_{\mathrm{t}}, B_{\mathrm{z}}\right.$, and the poloidal flux $\left.\Psi_{\mathrm{pol}}\right)$. For the moment toroidally symmetric equilibria have to be assumed. An exception to the assumption of toroidal symmetry is the magnetic field ripple. For ASDEX Upgrade an already established parametrization is used for the ripple [59]. It should be noted that ECRad could be easily extended to three-dimensional equilibria, since the only missing component are routines for the interpolation of three-dimensional equilibria.

Additional to the equilibrium, the $T_{\mathrm{e}}$ and $n_{\mathrm{e}}$ profiles, the antenna pattern of the ECE diagnostic and a contour of the vessel wall are needed to compute $T_{\text {rad. }}$ The configuration of ECRad is controlled through an input file. These 
settings include whether thermal or non-thermal distributions are considered, whether X- or/and O-mode should be assumed and how the IF bandwidth of the radiometer and the volume of sight are to be discretized. If ECRad is used as a library all the above quantities are provided through interface functions.

At present the ECRad library is implemented for thermal plasmas only, where $T_{\mathrm{e}}$ and $n_{\mathrm{e}}$ are assumed to be flux functions. In the stand-alone version $T_{\mathrm{e}}$ and $n_{\mathrm{e}}$ can be chosen to be two-dimensional matrices using the same grid as the magnetic equilibrium. For the study of non-thermal ECE the stand-alone version allows one to supply a distribution function profile. The distribution function has to be provided on a grid spanned by $\rho_{\text {pol }}$, normalized momentum $u$ and pitch angle $\zeta$

$$
u=\sqrt{u_{\perp}^{2}+u_{\|}^{2}}, \quad \zeta=\arccos \left(\frac{\sqrt{u_{\|}^{2}+\frac{\xi-1}{\xi} u_{\perp}^{2}}}{u}\right), \quad \xi \equiv \frac{|\boldsymbol{B}|}{B_{\min }\left(\rho_{\mathrm{pol}}\right)},
$$

where $B_{\min }\left(\rho_{\text {pol }}\right)$ is the lowest magnetic field strength on a flux surface.

For a detailed list on all the ECRad settings please see table C.4.

\subsection{Interpolation}

Several of the input quantities provided to ECRad need to be interpolated.

\subsubsection{One-dimensional and two-dimensional interpolation}

The one-dimensional profiles $T_{\mathrm{e}}, n_{\mathrm{e}}$ and $B_{\mathrm{min}}$ are interpolated with the univariate spline provided by the FITPACK package [60]. To avoid negative values for $n_{\mathrm{e}}$ and $T_{\mathrm{e}}$, their natural logarithm is interpolated. For library usage it is possible to provide ECRad with bindings to customized functions for the $T_{\mathrm{e}}$ and $n_{\mathrm{e}}$ evaluation. This allows a consistent treatment of $T_{\mathrm{e}}$ and $n_{\mathrm{e}}$ across multiple forward models in case of multi-diagnostic data analysis. Two-dimensional quantities, i.e. the three components of $\boldsymbol{B}$, the flux matrix and $T_{\mathrm{e}}$ and $n_{\mathrm{e}}$ if they are provided as matrices, are interpolated with the rectangular bivariate spline provided by the FITPACK [60] package.

\subsubsection{Interpolation of numerical distribution function profiles}

The three-dimensional distribution function profile $\left(\rho_{\text {pol }}, u\right.$ and $\left.\zeta\right)$ has to be interpolated in the radial direction $\left(\rho_{\text {pol }}\right)$ and in spherical momentum space. Furthermore, the derivatives in $u_{\perp}$ and $u_{\|}$of the distribution function are needed to evaluate the absorption coefficient. The interpolation and the differentiation in momentum space is achieved with the rectangular bivariate spline from the FITPACK package [60]. The radial interpolation of the distribution function is handled by a univariate spline interpolation of each momentum space grid cell. Equivalent to the interpolation of $T_{\mathrm{e}}$ and $n_{\mathrm{e}}$ the natural logarithm of the distribution function is interpolated. This ensures that the values to be interpolated do not vary over several orders of magnitude and that the distribution function is always positive. Zero values in the numerical distributions are replaced by a small positive number $\left(1.0 \times 10^{-30}\right)$. 


\subsection{Antenna pattern}

Like in the model of Ref. [4], the volume of sight of each ECE antenna is discretized into a rectangular bundle of $N_{\text {ray }}$ rays. The number of rays has to be either one or $N_{\text {ray }}=N^{2}+1$ with integer $N \geq 2$ or $N=0$ for a single ray per channel. The +1 is due to the central ray, which is mandatory. Even though it only enters the computation of $T_{\text {rad }}$ if $N_{\text {ray }}=1$, it is needed to map quantities like the emissivity or the absorption coefficient from the individual rays to a single LOS. It is assumed that the volume of sight of the diagnostic is a Gaussian beam with the $1 / e$ beam width $w$ of the electric field. The rays are spanned on a grid suitable for Gaussian quadrature.

One of the limitations of geometrical optics is that diffraction is neglected and the beam waist is zero at the focus point. To mitigate this, the initial conditions of the rays are chosen such that the beam width $w$ at the focus point $s_{\text {focus }}$ equals the beam width expected of a Gaussian beam in vacuum. The evolution of the beam width of a Gaussian beam in vacuum is given by [61]

$$
w(s)=w_{0} \sqrt{1+\frac{\lambda^{2}\left(s-s_{\text {waist }}\right)^{2}}{\left.\pi^{2} w_{0}^{4}\right)},}
$$

with $s$ the arc length of the beam, $\lambda$ the wave length, $s_{\text {waist }}$ the position of the waist and $w_{0}$ the beam waist. Both $w_{0}$ and $s_{\text {waist }}$ are calculated from the radius of curvature $R_{\text {curv }}$ and the beam width at the antenna. The evolution of $R_{\text {curv }}(s)$ of a Gaussian beam in vacuum is given by

$$
R_{\text {curv }}(s)=\left(s_{\text {waist }}-s\right)\left(1+\frac{\pi^{2} w_{0}^{4}}{\lambda^{2}\left(s-s_{\text {waist }}\right)^{2}}\right) .
$$

The discretization of frequency bandwidth is also adapted from Ref. [4] and, similar to the number of rays $N_{\text {freq }}$ has to be odd such that a central frequency exists. The bandwidth is only considered for the radiation transport and only the central frequency enters the geometrical optics equations to minimize computational time. For the averaging of $T_{\text {rad }}$ over the bandwidth a Gaussian quadrature algorithm is used.

The code expects that the provided antenna position is sufficiently far away from the confined region of the plasma such that $n_{\mathrm{e}}$ at the antenna position is negligibly small. It is not required that this position lies inside the domain where the magnetic equilibrium and the input $T_{\mathrm{e}}$ and $n_{\mathrm{e}}$ profiles are defined. In this case vacuum propagation is assumed until a position inside the domain is reached.

\subsection{Ray tracing}

The geometrical optics equations are solved using the DLSODE [62] package. While the code assumes a toroidally symmetric equilibrium, the toroidal inhomogeneity due to the magnetic field ripple is included in the ray tracing. The chain rule is applied to compute the right-hand side of equation (1) analytically for improved numerical stability. Straight propagation is assumed until a 
position is reached, where the magnetic equilibrium and the kinetic profiles are defined and $\rho_{\text {pol }}<1.20$, or $\left(\frac{\omega_{\mathrm{p}}}{\omega}\right)^{2}>0.04$ with $\omega_{\mathrm{p}}$ the plasma frequency. Additionally to these conditions it is necessary that the position lies inside the vessel contour. Upon plasma entry the rays are coupled to the plasma by Snell's law. The propagation is stopped if either the ray leaves the vessel contour, reaches the upper hybrid resonance $\omega_{\mathrm{UH}}^{2}=\omega_{\mathrm{p}}^{2}+\omega_{\mathrm{c}}^{2}$ in case of X-mode polarization, or if it leaves the domain for which the magnetic equilibrium or the kinetic profiles are defined.

Another noteworthy exit condition for the ray tracing algorithm are reflections at cut-off layers inside the plasma [17]. If the wave vector $\boldsymbol{k}(s)$ deviates by more than $90^{\circ}$ from the initial wave vector $\boldsymbol{k}\left(s_{\text {ant }}\right)$ the ray tracing is also stopped. The reason for this approach are uncertainty issues. For flat $n_{\mathrm{e}}$ profiles, as often encountered in the core of tokamak plasmas, even a small uncertainty in $n_{\mathrm{e}}$ or the magnetic equilibrium can give rise to large errors in the ray path near and especially after a cut-off layer is encountered. Accordingly, the amount of ECE that is scattered into the antenna by the cut-off layer is subject to large uncertainties. To circumvent the computational effort necessary to accurately propagate the uncertainty of the density profile and the magnetic equilibrium onto the prediction of $T_{\text {rad }}$ in a cut-off situation the treatment above was chosen. Since the ray path is shortened by this approach, the predicted optical depth is lower than it would be for the full path. If the optical depth of the shortened path is low, the measurement can be removed automatically from the data analysis according to the criterion described in section 3.8. If, however, the measurement is optically thick even for the shortened ray path the amount of radiation scattered off the cut-off layer and toward the antenna is irrelevant, because it is reabsorbed. Hence, unnecessary computational effort is avoided.

\subsection{Radiation transport}

The radiation transport equation, equation (7), can be solved by a simple, explicit 4th order Runge-Kutta scheme on a static grid. For the spacing of the grid the approach of Ref. [4] was adapted. Accordingly, dense grids near resonances are nested into a sparse grid that covers the remaining LOS. Since the grid is static it is possible that the combination of strong absorption with an insufficiently small grid size leads to large numerical errors, which can give rise to negative radiation temperatures. Such cases are automatically identified by ECRad and are resolved with one of two strategies. If the distribution function is thermal and the total optical depth $\tau_{\omega}$ of the current grid cell is so large that it is optically thick (adjustable, default: $\tau_{\omega}>5$ ) then the radiation temperature is set to the electron temperature of the grid cell and the computation continues. In case of non-thermal distribution functions or insufficiently large optical depth of the cell, the grid is recomputed using half of the initial cell size. Smaller numerical errors are identified by comparing $T_{\text {rad }}$ obtained by the 4 th order Runge-Kutta scheme with $T_{\text {rad }}$ obtained with an explicit forward Euler scheme at each step. If the deviation between the two schemes is larger than $50 \%$ then the grid is also recomputed with a two times higher resolution. To assure that 
$T_{\text {rad }}$ is continuously differentiable with respect to the $T_{\text {e }}$ profile, changes of the grid size must not be carried out during an optimization process. Because of this, it is useful to restart the inference of $T_{\mathrm{e}}$ multiple times, such that the grid can be adapted in between subsequent optimizations (see section 4.1).

\subsection{Absorption and emission}

The evaluation of the absorption coefficient and the emissivity (cf. equations (9) and (10)) is the most computationally intensive step when solving the radiation transport equation. For thermal plasmas it is sufficient to only evaluate the absorption coefficient and compute the emissivity via Kirchhoff's law. Far off resonance and for sufficiently small $T_{\mathrm{e}}$ the absorption coefficient and the emissivity are negligibly small. To identify these regions for thermal plasmas, a fast approximation formula was developed for the absorption coefficient. The details of this approximation can be found in appendix Appendix B. By using the approximation to check if the absorption coefficient needs to be evaluated, the total amount of computations of the absorption coefficient is reduced by 30 to $60 \%$ depending on the plasma scenario.

\subsection{Wall reflections}

For the lower range of measured frequencies, for which the cold resonances lie near the plasma edge or in the scrape-off layer (SOL), the optical depth of the plasma $\tau_{\omega}$ can be very small. This means that the radiation passes through the plasma multiple times due to wall reflections without being fully absorbed. The amount of radiation scattered into the antenna by reflections on the vessel wall can be estimated by an infinite reflection model [4,63], if the optical depth of the measurement is not too low [63]. The model assumes radial propagation between plane-parallel walls, such that neither the trajectory of the wave nor its polarization is affected by the reflection. The implementation in ECRad allows for independent wall reflection coefficients for X- and O-mode. Furthermore, it is possible to consider that wall reflections converts a user defined fraction of O-mode to X-mode.

In practice this approach produces satisfactory results if $\tau_{\omega}>1$. At very low optical depths $\left(\tau_{\omega} \lesssim 0.5\right)$ the simple model of infinite reflections is insufficient and the agreement between forward modeled $T_{\text {rad }}$ and the measurements can become quite poor (see ref. [32]). For this reason the ECRad model is not expected to be reliable if the optical depth of a measurement is below $\tau_{\omega} \lesssim 0.5$.

The scaling factor $\kappa_{\text {Ref }}$ between the observed intensity $I_{\omega, \text { Refl }}$ and the intensity obtained from the radiation transport $I_{\omega}$ is given by [63]:

$$
\kappa_{\text {Refl }} \equiv \frac{I_{\omega, \text { Refl }}}{I_{\omega}}=\frac{1}{1-R_{\text {wall }} \mathrm{e}^{-\tau_{\omega}}}
$$

The wall reflection coefficient is given by $R_{\text {wall }} \in[0,1] . \quad R_{\text {wall }}$ is not simply a material property, Because of the simplicity of the infinite wall reflection model. It also characterizes the average $T_{\text {rad }}$ that each consecutive pass through the 
plasma provides. Note that in practice this is not simply the $T_{\text {rad }}$ that the initial pass provided, since each reflection will produce a unique LOS.

As shown in Ref. [4] values close to one show the best fit for a majority of the measurements. In the ECRad code the wall reflection coefficients for X-and O-mode are treated individually. The default values for $R_{\text {wall }}$ is 0.90 for both, the $\mathrm{X}$-mode and the O-mode. For the X-mode the value is adopted from Ref. [4]. For the O-mode this value is arbitrarily chosen, as O-mode emission has not been interpreted successfully with ECRad yet [35]. Per default the scaling factor $\kappa_{\text {Refl }}$ is only considered for measured frequencies with $\tau_{\omega}<9$.

\subsection{The polarization filter}

Most ECE diagnostics have a beam-splitting polarizer designed to filter either X- or O-mode radiation. In case of a radial ECE diagnostic and if X-mode is desired, the wires of the filter are usually aligned with the toroidal direction of the torus. Because of the poloidal magnetic field and to some extent due to the magnetic field ripple this implies that the filter is not perfectly aligned with $\boldsymbol{B}$, even if the LOS is perfectly radial. The ECRad code allows polarization mismatch to be considered in the calculation of $T_{\text {rad }}$. The modeled $T_{\mathrm{rad}}$ is then given by:

$$
T_{\mathrm{rad}, \bmod }=\left(\boldsymbol{e}_{\mathrm{X}} \cdot \boldsymbol{p}\right)^{2} T_{\mathrm{rad}, \bmod , \mathrm{X}}+\left(\boldsymbol{e}_{\mathrm{O}} \cdot \boldsymbol{p}\right)^{2} T_{\mathrm{rad}, \bmod , \mathrm{O}}
$$

The ray paths and radiation temperatures for the $\mathrm{X}$ - and O-mode are calculated independently of each other. The fractions of X- and O-mode that pass through the filter are obtained by projecting the (normalized) polarization vector $\boldsymbol{e}_{\mathrm{X} / \mathrm{O}}$ of the $\mathrm{X} / \mathrm{O}$-mode onto the polarization vector of the filter $\boldsymbol{p}$. The polarization vector is calculated for the plasma parameters at the last closed flux surface. This practice is common for the determination of electron cyclotron resonance heating (ECRH) polarization and it is expected to be also appropriate for ECE [64]. Cold plasma dispersion is considered for the computation of $\boldsymbol{e}_{\mathrm{X} / \mathrm{O}}$ $[40,52]$.

\subsection{Output}

The output provided by ECRad depends on whether it is used as a library or run stand-alone. In the latter case the code has two primary modes of operation. One is designed for speed, which produces solely the cold resonance positions, $T_{\text {rad }}$ and $\tau_{\omega}$ as output. The other mode is designed for highest verbosity. In this mode the radiation transport equation is always solved twice considering two sets of absorption coefficients and emissivities. For thermal plasmas the primary set of $T_{\mathrm{rad}}$ is obtained with equations (9) and (14). The secondary set of $T_{\mathrm{rad}}$ considers the absorption coefficient and emissivity according to Ref. [48] and Kirchhoff's law. The approach from Ref. [48] features higher overall accuracy, because the absorption coefficient is derived self-consistently from the fully relativistic dispersion relation. This means that it is also appropriate for ECE of the fundamental resonance, unlike the primary model. These two features come at increased computational cost and at reduced robustness. The purpose of the 
secondary set of $T_{\text {rad }}$ is to allow the identification of cases, where equation (9) is inappropriate. In case of non-thermal distributions the primary and secondary results are obtained with equations (9) and (10). The primary results consider the non-thermal distributions, while thermal equilibrium is assumed for the secondary results. In verbose mode ECRad produces the birthplace distribution of observed intensity binned to a signed $\rho_{\text {pol }}$ axis, where negative signs correspond to positions on the HFS. It also provides warm resonance positions and the birthplace distributions for each considered ray which allows the birthplace distribution to be fully resolved in all three spatial coordinates. For the details on the output files generated by the graphical user interface (GUI) see appendix Appendix C.

The ECRad library provides an interface function that delivers $T_{\mathrm{rad}}$ for thermal plasmas as computed by the primary model. Additionally, an interface function exists which retrieves the birthplace distribution for a list of ECE channels as specified by the user.

\subsection{Graphical User Interface}

A GUI has been developed for computations with ECRad. It is written in python 2.7 and uses the wx-Library[65] as a front-end. The GUI features the automatic loading of profiles from the ASDEX Upgrade database, controlling the ECRad configuration and antenna pattern of the ECE diagnostic. It is not tied to the ASDEX Upgrade environment and all the information required by ECRad can also be provided to the GUI through MATLAB data files. To quickly examine the results from ECRad the GUI allows $T_{\text {rad }}, \tau_{\omega}$, the birthplace distributions and the ray geometry to be visualized. Lastly the GUI has tools to perform and examine cross-calibrations of ECE diagnostics (cf. section 4.2).

\section{Example applications}

In this section two applications of ECRad are discussed. The first case shows how ECRad is used for the inference of $T_{\mathrm{e}}$ with the IDA used at ASDEX Upgrade. Several examples for the application of IDA and ECRad can be found in Ref. [32]. Most noteworthy are the successful treatment of the optically gray region near the plasma edge and the interpretation of ECE measurements affected by harmonic overlap. As a new application of ECRad we present in section 4.2, how an oblique ECE diagnostic can be cross calibrated against a known $T_{\text {e }}$ profile. ECRad has also been used extensively to compare the state of the art theory for electron cyclotron damping against measurements. The detailed report on this work will be subject to a future paper. First results can be found in Ref. [37].

\subsection{ECRad in IDA}

ECRad has been used as the ECE forward model in IDA for several years. The strategy of ECRad's usage has evolved and several tweaks were found that 
significantly reduce the run time. In this section the implementation of ECRad in IDA is discussed.

IDA is a Bayesian data analysis tool kit in which the $n_{\mathrm{e}}$ and $T_{\mathrm{e}}$ profiles are determined simultaneously from a data set comprised of the measurements of multiple diagnostics [31]. The goal of the analysis is to find the maximum posterior. In IDA this multi-dimensional, non-linear optimization problem is split into five, subsequent optimizations, where the result of the previous optimization provides the starting point for the subsequent optimization.

In the first optimization only classical ECE is considered. Since ECRad is the most expensive forward model in IDA, falling back on classical ECE analysis significantly reduces the computational cost of the first iteration. Before the first optimization step it is necessary to identify any measurement that is beyond the scope of classical ECE analysis. An example would be a channel affected by harmonic overlap (see Ref. [32]). Once the first sub-optimization is completed the following steps are performed before each of the four remaining sub-optimizations:

$\rightarrow$ Perform ray tracing for all ECE channels.

$\rightarrow$ Obtain the cold resonance positions along the refracted LOS as they are needed for classical ECE analysis.

$\rightarrow$ Determine if the amount of points in the numerical integration of the absorption coefficient (cf. equation (10)) can be reduced or needs to be increased.

$\rightarrow$ If the current estimates for the $T_{\mathrm{e}}$ and $n_{\mathrm{e}}$ profile cause the grid of the radiation transport equation to be too coarse, ECRad will automatically switch to a finer grid every time it is called in between the sub-optimization calls.

$\rightarrow$ Use the optical depth predicted by ECRad to remove any channel strongly affected by wall reflections $\left(\tau_{\omega} \leq 0.5\right)$ from the considered data set.

$\rightarrow$ Select all channels with intermediate optical depths $0.5<\tau_{\omega}<5$ and flag them as "requires ECRad".

$\rightarrow$ Compare result from classical ECE analysis with synthetic $T_{\text {rad }}$ for remaining channels. Flag all channels with more than a $2 \%$ relative deviation between the two estimates as "requires ECRad".

$\rightarrow$ Only use ECRad where necessary. Fallback to classical ECE analysis for all other channels.

One of the most important performance improvements is to keep the number of channels that are forward modeled as low as possible. Another important improvement is to perform ray tracing only in between sub-optimizations. Strictly speaking a fully self-consistent treatment would require ray tracing every time 
$T_{\text {rad }}$ is evaluated. However, recalculating the ray trajectory in each optimization step is not feasible for two reasons. First, computing the ray trajectories is much more expensive than solving the radiation transport equation. Second, as explained earlier, it is possible that a large change in $n_{\mathrm{e}}$ during the optimization causes ECE channels to enter cut-off, and, consequently, leave the domain in which ECRad predicts $T_{\text {rad }}$ accurately (see Ref. [35]). A solution would be to remove the affected ECE channels from the analysis but altering the set of considered measurements during an optimization causes a failure of the optimization routine. To avoid such situations, the $n_{\mathrm{e}}$ profile provided to ECRad by IDA is frozen to the results obtained from the previous sub-optimization for all but the last sub-optimization.

In practice the inability to perform ray tracing for each computation of $T_{\text {rad }}$ is unproblematic. In case of the profile radiometer at ASDEX Upgrade, the influence of refraction on the measurements is weak. Hence, ray tracing only in between optimization steps in IDA is sufficient. Five sub-optimization steps are observed to be sufficient for obtaining converged results in routine data analysis. For applications of radiation transport modeling in the analysis of ECE measurements see also Refs. [4, 32].

\subsection{Cross calibration of oblique ECE diagnostics}

The typical hot-cold-calibration technique [66] might not be possible for all ECE diagnostics due to technical reasons, like, e.g., the lack of temperature control of the radiometer causing the calibration factors to drift slowly over the day, or the lack of room for an in-situ calibration setup. Nevertheless, measurements of $T_{\text {rad }}$ can still be obtained by cross-calibrating the diagnostic against known $T_{\text {e }}$ profiles. For example, the ASDEX Upgrade collective Thomson scattering system[67] can be used as an uncalibrated, steerable, oblique ECE diagnostic for the study of non-thermal electron distribution functions. For oblique ECE diagnostics classical ECE analysis is inappropriate because the LOS can be subject to strong refraction and the emission is broadened by the Doppler-effect. Hence, radiation transport modeling is required for the cross-calibration. In the following the details of the cross-calibration method implemented in the python GUI of ECRad are explained in section 4.2.1. Additionally, an example of a cross-calibration is shown.

\subsubsection{Cross-Calibration method}

The goal of the cross-calibration is to obtain a calibration coefficient $C$ and its uncertainty for each channel of the diagnostic. For this purpose a linear regression is performed

$$
V_{\mathrm{i}, \mathrm{diag}}^{*}(t)=Q_{i} T_{i, \mathrm{rad}, \bmod }(t)+V_{i, 0}^{*},
$$

where $V_{\mathrm{i}, \text { diag }}^{*}$ is the preprocessed signal measured by the channel $i$ of the diagnostic, $Q_{i}=1 / C_{i}$ the inverse of the cross-calibration coefficient, $T_{i, \text { rad,mod }}(t)$ the forward modeled $T_{\text {rad }}$ given the plasma parameters at time $t$, and $V_{\mathrm{i}, 0}^{*}$ the 
offset of the regression. The preprocessing consists of three steps. Most radiometers have an offset, i.e. even if there is no plasma the signal is non-zero. The first step is to remove the offset by subtracting the average signal level in the time window from 0 to $1 \mathrm{~ms}$. The length of this window is adjustable in the GUI. After the offset removal, the signal is median filtered with a kernel/window size of 3 to remove potential outliers. In the third step the signal is averaged. The statistical uncertainty of $V_{i, \text { diag }}^{*}$ is determined by computing the standard deviation of the median filtered signal. The linear regression yields $Q_{i}$, the associated statistical uncertainty $\Delta Q_{i, \text { stat }}, V_{i, 0}^{*}$ and the corresponding statistical uncertainty $\Delta V_{i, 0, \text { stat }}^{*}$. The statistical uncertainty of the cross-calibration factor $C_{i}$ is computed from $\Delta Q_{i, \text { stat }}$ using Gaussian error propagation.

The systematic uncertainty $\Delta C_{i, \mathrm{sys}}$ is approximated by computing the standard deviation of the time resolved calibration coefficient $c_{i}(t) \equiv T_{i, \text { rad,mod }}(t) / V_{i, \text { diag }}^{*}(t)$.

\subsubsection{Example}

At ASDEX Upgrade the collective Thomson scattering system [67] allows oblique ECE measurements. In the $-1.8 \mathrm{~T}$ discharge \#35662, both receivers of the collective Thomson scattering system, "CTA" and "CTC", were used to measure the ECE around $105 \mathrm{GHz}$, which corresponds to cold resonance positions at around $\rho_{\text {pol,res }} \approx 0.2$. The toroidal angles of the two receivers were chosen such that one receiver was sensitive to co-streaming electrons, i.e. $\phi_{\text {tor }}=$ $-20^{\circ}$ for TORBEAM convention[42], and the other $\left(\phi_{\text {tor }}=20^{\circ}\right)$ to counterstreaming electrons with respect to the plasma current. For both receivers the polarizers were set to X-mode and $100 \% \mathrm{X}$-mode is considered in the analysis.

Figure 3 summarizes the cross-calibration process. Figure a) depicts the temporal evolution of $c_{40}(t)$ and $T_{40, \mathrm{rad} \text {,mod }}$ for channel 40 of receiver "CTA", with $f=106.55 \mathrm{GHz} . \quad c_{40}(t)$ differs at most about $5 \%$ from the average. This is very close to the ideal case of a constant calibration factor. Note that all the plasma parameters entering the cross-calibration, i.e. $T_{\mathrm{e}}, n_{\mathrm{e}}$ and the magnetic equilibrium are derived from measurements and are, therefore, uncertain. Figure $3 \mathrm{~b}$ ) shows the heating power of the Neutral Beam Injection (NBI) system and $n_{\mathrm{e}}$ at the magnetic axis. The NBI heating was modulated such that it performed a triangular shaped power scan. This results in a variation of $T_{\mathrm{e}}$, which is necessary to get adequate estimates of the systematic uncertainties. Figure c) shows the linear regression. The results of the cross-calibration are: $\left|C_{40}\right|=33.9 \mathrm{keV} \mathrm{V}^{-1}, \Delta C_{40, \text { stat }}=0.16 \mathrm{keV} \mathrm{V}^{-1}, \Delta C_{40, \mathrm{sys}}=0.9 \mathrm{keV} \mathrm{V}^{-1}$ and $V_{40,0}^{*}=0.2 \mathrm{mV}, \Delta V_{40,0, \text { stat }}^{*}=0.2 \mathrm{mV}$. This corresponds to a total uncertainty of the cross-calibration of well below five percent.

\section{Conclusions}

The code ECRad for the interpretation of ECE data was presented. ECRad has been used extensively for the interpretation of ECE measurements at ASDEX Upgrade [31, 32, 33, 68]. Large scale data analysis is made feasible by ECRad's low computational cost, high robustness and the support for gradient based 

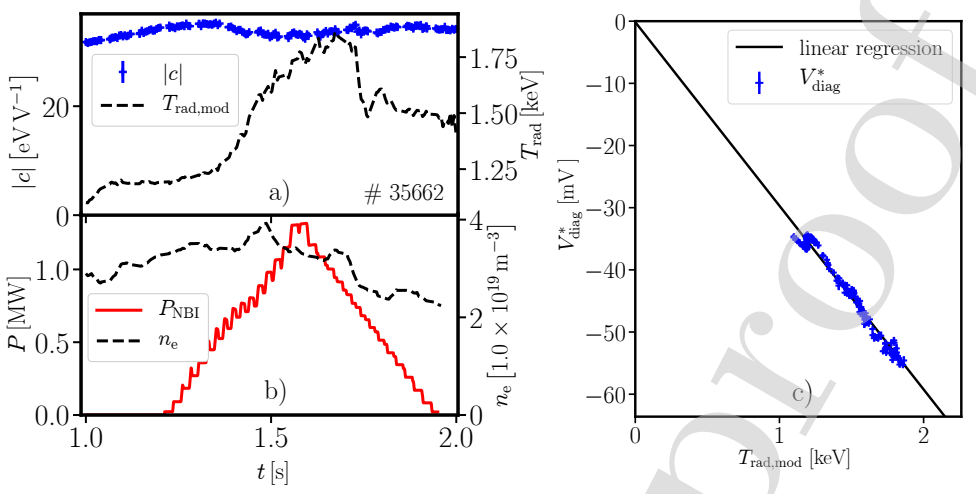

Figure 3: Summary of the cross calibration for discharge \# 35662 and channel 40 of the "CTA" receiver. a) The time trace of the absolute value of the time resolved calibration coefficient $c_{40}(t)$ (left y-axis) and the forward modeled $T_{\text {rad,mod. }}$ b) NBI heating power (left $\mathrm{y}$-axis) and $n_{\mathrm{e}}$ at the magnetic axis (right y-axis) as functions of time. c) the linear regression of the measured signal $V_{\text {diag }}^{*}$ vs. $T_{\text {rad,mod }}$.

optimizers. Additionally, it is also capable of predicting ECE spectra for arbitrary electron distribution functions. The key to achieve this is the absorption coefficient given by Ref. [52]. Supplementing this absorption coefficient with geometrical optics ray tracing allows the interpretation of oblique ECE measurements with ECRad. Two example applications of ECRad, the implementation of ECRad in IDA and the cross-calibration of oblique ECE diagnostics against known $T_{\mathrm{e}}$ profiles were discussed. The revisions of the code are managed through a git repository. This paper describes the revision with the tag " 1.0 ". The code is distributed by Rainer Fischer (rainer.fischer@ipp.mpg.de) upon request, after a software agreement with the Max-Planck-Institut für Plasmaphysik, excluding the commercial exploitation of the code, has been signed.

\section{Acknowledgements}

"This work has been carried out within the framework of the EUROfusion Consortium and has received funding from the Euratom research and training programme 2014-2018 and 2019-2020 under grant agreement No 633053. The views and opinions expressed herein do not necessarily reflect those of the European Commission."

\section{Appendix A. Emissivity}

While the absorption coefficient of ECRad can be adopted directly from Ref. [52], a rigorous derivation of the emissivity given by equation (9) cannot 
be found in the literature and it is, therefore, provided by this appendix. The starting point is equation (2.9) of Ref. [38].

$$
j_{\omega}=N_{\omega, \text { ray }}^{2} \frac{\omega^{2}}{2 \pi^{2} c_{0}^{2}\left|\boldsymbol{v}_{\mathrm{g}}\right|}\left|\omega \frac{\partial \hat{H}}{\partial \omega}\right|^{-1} \boldsymbol{e}^{*} \cdot\left\langle\boldsymbol{j} \boldsymbol{j}^{*}\right\rangle \cdot \boldsymbol{e},
$$

where $\langle\boldsymbol{j} \boldsymbol{j} *\rangle$ is given by equation (2.11a) of Ref. [38]

$$
\langle\boldsymbol{j} \boldsymbol{j} *\rangle=\frac{m_{\mathrm{e}, 0} c_{0}^{2} \omega_{p}^{2}}{2 \omega} \sum_{n} \int_{\boldsymbol{R}^{3}} \frac{d^{3} u}{\gamma} \delta\left(\gamma-N_{\|} u_{\|}-\frac{n \omega_{\mathrm{c}, 0}}{\omega}\right) \boldsymbol{V}_{n} \boldsymbol{V}_{n}^{*} f(\boldsymbol{u})
$$

and $\boldsymbol{V}_{n}$ by equation (2.11b) of Ref. [38]

$$
\boldsymbol{V}_{n}=\left(u_{\perp} \frac{n}{b_{n}} J_{\mathrm{n}}\left(b_{n}\right),+i u_{\perp} J_{\mathrm{n}}^{\prime}\left(b_{n}\right), u_{\|} J_{\mathrm{n}}\left(b_{n}\right)\right) .
$$

The three equations were adjusted such that the notation is consistent with the remaining paper. All quantities used in equations (A.1) to (A.3) were already introduced in section 2.3 except for the group velocity $\boldsymbol{v}_{\mathrm{g}}$, the geometrical optics Hamiltonian $\hat{H}$ and the normalized electric field, or polarization vector, $\boldsymbol{e}=$ $\boldsymbol{E} /|\boldsymbol{E}|$.

To obtain equation (9), equation (A.1) has to be expressed in terms of $\tilde{\boldsymbol{e}}$ (cf. equation (11)). To do so the absolute value of the Poynting flux in the geometrical optics limit is needed

$$
|S|=\left|\boldsymbol{v}_{\mathrm{g}}\right|\left|\omega \frac{\partial \hat{H}}{\partial \omega}\right| \frac{|\boldsymbol{E}|^{2}}{8 \pi}
$$

From this one can deduce

$$
\boldsymbol{e}^{*} \cdot\left\langle\boldsymbol{j} \boldsymbol{j}^{*}\right\rangle \cdot \boldsymbol{e}=\frac{\left|\boldsymbol{v}_{\mathrm{g}}\right|}{2 c_{0}}\left|\omega \frac{\partial \hat{H}}{\partial \omega}\right| \tilde{\boldsymbol{e}}^{*} \cdot\left\langle\boldsymbol{j} \boldsymbol{j}^{*}\right\rangle \cdot \tilde{\boldsymbol{e}}
$$

Inserting this expression into equation (A.1) produces equation (9) once it is simplified and transformed to cylindrical coordinates.

\section{Appendix B. Upper limit estimate for the absorption coefficient}

The upper-limit estimate is based on the approach given by Hutchinson [50], which uses the following approximations:

- The electrons are thermally distributed.

- The refractive index is $N_{\omega}=1$.

- The propagation is quasi-perpendicular. 
- The "polarization factor" is approximated in the non-relativistic limit. Hence, all factors $\gamma \approx 1$, which implies that $\beta_{\perp / \|}=u_{\perp / \|}$. Of course to have any relativistic broadening this approximation must not be applied to the resonance condition, where $\gamma$ is fully retained.

The two differences with respect to the treatment given by Hutchinson are that the fully relativistic distribution function is retained and that dimensionless momentum is used as the coordinate system. In the following expression the integration over $u_{\perp}$ is already carried out:

$$
\begin{aligned}
& \alpha_{\omega, n, \text { approx }} \approx \alpha_{0} \int_{u_{\|,-}}^{u_{\|,+}} f\left(u_{\perp}\left(u_{\|}\right), u_{\|}\right)\left(\frac{u_{\perp}\left(u_{\|}\right)}{2}\right)^{2 n+1} \mathrm{~d} u_{\|} \\
& \alpha_{0} \equiv \frac{\left(e \omega_{\mathrm{c}, 0}\right)^{2} n_{\mathrm{e}}}{8 \pi \epsilon_{0} c_{0}} \frac{n^{2(n-1)}}{(m-1) !}(\sin \theta)^{2 m-1}\left(\cos ^{2} \theta+1\right) \\
& u_{\perp}\left(u_{\|}\right) \equiv \sqrt{\left(\frac{n}{\bar{\omega}}-\cos \theta\right)^{2}-u_{\|}^{2}-1} \\
& u_{\|, \pm}=\frac{\frac{n}{\bar{\omega}} \cos \theta \pm \sqrt{\frac{n^{2}}{\bar{\omega}^{2}}+\cos ^{2} \theta-1}}{1-\cos ^{2} \theta}
\end{aligned}
$$

Since the distribution function can be expressed as a simple exponential function in $u_{\|}$the equation above has an analytical solution that can be obtained by repeatedly applying integration by parts. Although the expression obtained after the integration in $u_{\|}$is quite lengthy, the computational cost is still small since numerical integration is not necessary and no Bessel function needs to be evaluated.

This formula works very well as an upper limit for the absorption coefficient. This is demonstrated in figure B.4, where the approximation (dashed lines) is compared to the absorption coefficient given by equation (10) (solid lines). For the comparison the two absorption coefficients are shown as functions of the cyclotron frequency $\omega_{c, 0}$ normalized with the measurement frequency $\omega$. Figure B.4 a) shows the two absorption coefficients for a measurement frequency $f=140 \mathrm{GHz}$ and $n_{\mathrm{e}}=10.0,8.0$ and $6.0 \times 10^{19} \mathrm{~m}^{-3}$. In figure B.4 b) $f=$ $105 \mathrm{GHz}$ and $n_{\mathrm{e}}=6.0,4.0$ and $2.0 \times 10^{19} \mathrm{~m}^{-3}$. For the low frequency case smaller densities are necessary to avoid cut-off. For these calculations $T_{\mathrm{e}}=8 \mathrm{keV}$ and $\theta=80^{\circ}$ are considered. For all the combinations of $n_{\mathrm{e}}, f$ and $\frac{\omega}{\omega_{\mathrm{c}, 0}}$ shown in equation (B.1) figure B.4 delivers an upper limit for the absorption coefficient given by equation (10).

The absorption coefficient given by equation (10) is only evaluated, if

$$
\tau_{\text {approx }}=2 \Delta s \sum_{n=2}^{3} \alpha_{\omega, n, \text { approx }}>\tau_{\text {crit }},
$$



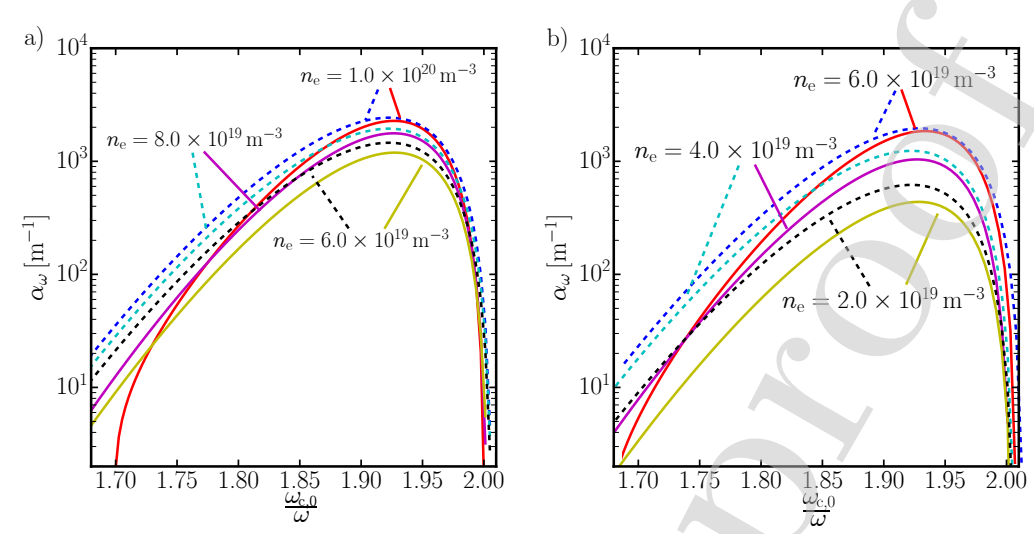

Figure B.4: The absorption coefficient given by equation (10) (sold lines) is compared to the absorption coefficient given by the crude approximation (cf. equation (B.1)) (dashed lines) for six $n_{\mathrm{e}}$ and two different measured frequencies $f_{\mathrm{ECE}}=140 \mathrm{GHz}$ (figure B. 4 a)) and $f_{\mathrm{ECE}}=105 \mathrm{GHz}$ (figure B.4 b)) as a function of the cyclotron frequency normalized to the measured frequency. For all frequency- and density combinations the upper limit estimation is larger than the absorption coefficient given by equation (10).

where $\Delta s$ is the local step size and $\tau_{\text {crit }}$ is an adjustable threshold. In practice a $\tau_{\text {crit }}=1.0 \times 10^{-8} \mathrm{~m}^{-1}$ has proven to not alter the forward modeled $T_{\text {rad }}$ of the test cases while improving the performance of the model considerably.

\section{Appendix C. Input and Output of ECRad}

At the time of this writing the output of ECRad is stored in a large amount of ASCII encoded files, which are not optimally organized. This is compensated by the GUI of ECRad that automatically reads the ASCII output and stores them as a single binary file using the Matlab [69] formatting ".mat". All fields in the ".mat" file are described below in tables C.1 to C.5.

If not otherwise noted all quantities are given in SI-units. The dimension of the individual quantities is noted in the table caption. For many quantities there is either a "_comp" or secondary counterpart. These quantities are derived from the secondary absorption coefficient, which is either the fully relativistic treatment in case of thermal plasmas, or according to a thermal distribution using equations 9 and 10 in case of non-thermal plasmas.

Tables C.1 to C.3 summaries the fields of the ".mat"-file that contain the results, where table C. 1 contains the main results, table C. 2 describes the details on the parameters on/of the individual rays and table C.3 lists the quantities related to a cross-calibration. The file also contains all information on the ECRad settings (see table C.4) and the considered scenario (see table C.5). 


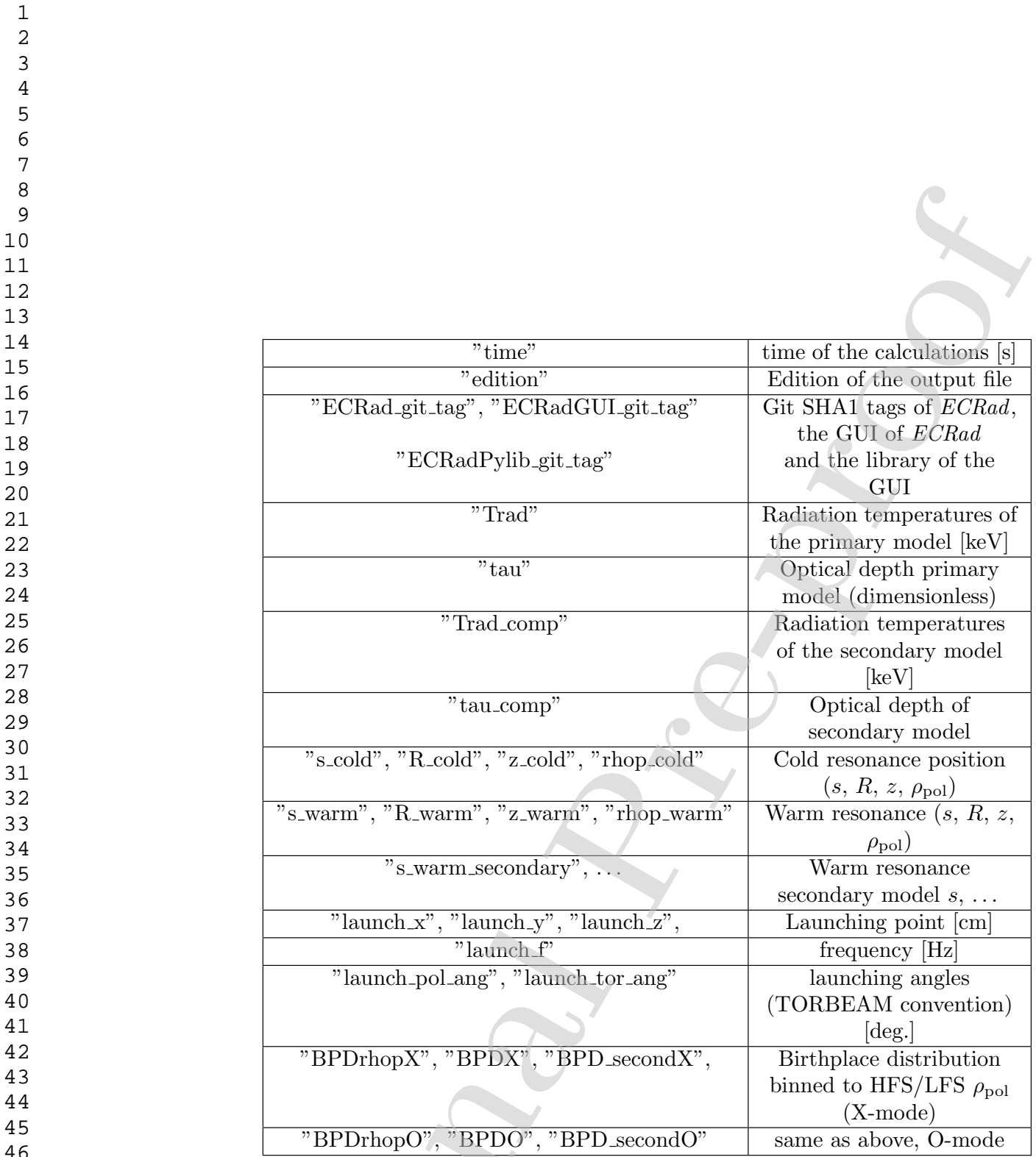

Table C.1: Main results of the ECRad calculation. With the exception of time, and edition, the git tags, and the birthplace distribution, all quantities have as first index time and as second index channel number. The birthplace distributions are 3 dimensional fields, where the third index is the $\rho_{\text {pol }}$ axis. 


\begin{tabular}{|c|c|}
\hline "sX", "xX", "yX", "zX","rhopX" & $\begin{array}{c}\text { LOS of each ray, } s, x, y, z, \rho_{\text {pol }}, \\
\text { X-mode }\end{array}$ \\
\hline "sO", "xO", ... & Same as above O-mode \\
\hline "HX" & $\begin{array}{l}\text { Hamiltonian from ray tracing, } \\
\text { X-mode. } \\
\text { Values greater than } 1.0 \times 10^{-4} \\
\text { indicate problems in the ray tracing. }\end{array}$ \\
\hline "HO" & Same as above, O-mode \\
\hline$" N X ", " N c X "$ & $\begin{array}{l}\text { Refractive index of the ray and } \\
\text { refractive index from the } \\
\text { Appleton-Hartree dispersion relation, } \\
\text { X-mode }\end{array}$ \\
\hline "NO", "NcO" & Same as above, O-mode \\
\hline "XX", "YX" & $X$ and $Y, \mathrm{X}$-mode \\
\hline "XO", "YO" & $X$ and $Y$, O-mode \\
\hline "thetaX" & $\theta \equiv \arccos \left(\frac{\boldsymbol{B} \cdot \boldsymbol{N}}{|\boldsymbol{B}||\boldsymbol{N}|}\right)[\mathrm{rad}], \mathrm{X}$-mode \\
\hline "thetaO" & Same as above O-mode \\
\hline "TeX" & $T_{\mathrm{e}}[\mathrm{eV}], \mathrm{X}$-mode \\
\hline "TeO" & $T_{\mathrm{e}}[\mathrm{eV}]$, O-mode \\
\hline "ray_BPDX", "ray_BH & $\begin{array}{l}\text { Birthplace distribution along ray, } \\
\text { primary and secondary model, } \\
\text { X-mode }\end{array}$ \\
\hline "ray_BPDO", "ray_BPD_secondO" & Same as above, O-mode \\
\hline "ray_emX", "ray_abX", "ray_TX" & $\begin{array}{l}\text { Emissivity, absorption coefficient and } \\
\text { transmitivity, primary model, X-mode }\end{array}$ \\
\hline "ray_em_secondX", & $\begin{array}{c}\text { Sames above, secondary model, } \\
\text { X-mode }\end{array}$ \\
\hline & $\begin{array}{l}\text { Emissivity, absorption coefficient and } \\
\text { transmitivity, primary model, O-mode }\end{array}$ \\
\hline "ray_em_secondO", & $\begin{array}{l}\text { Sames above, secondary model, } \\
\text { O-mode }\end{array}$ \\
\hline
\end{tabular}

Table C.2: Quantities computed on each ray. Accordingly, each field is three dimensional if a single ray is considered per channel, or four dimensional in case of more than a single ray per channel. 


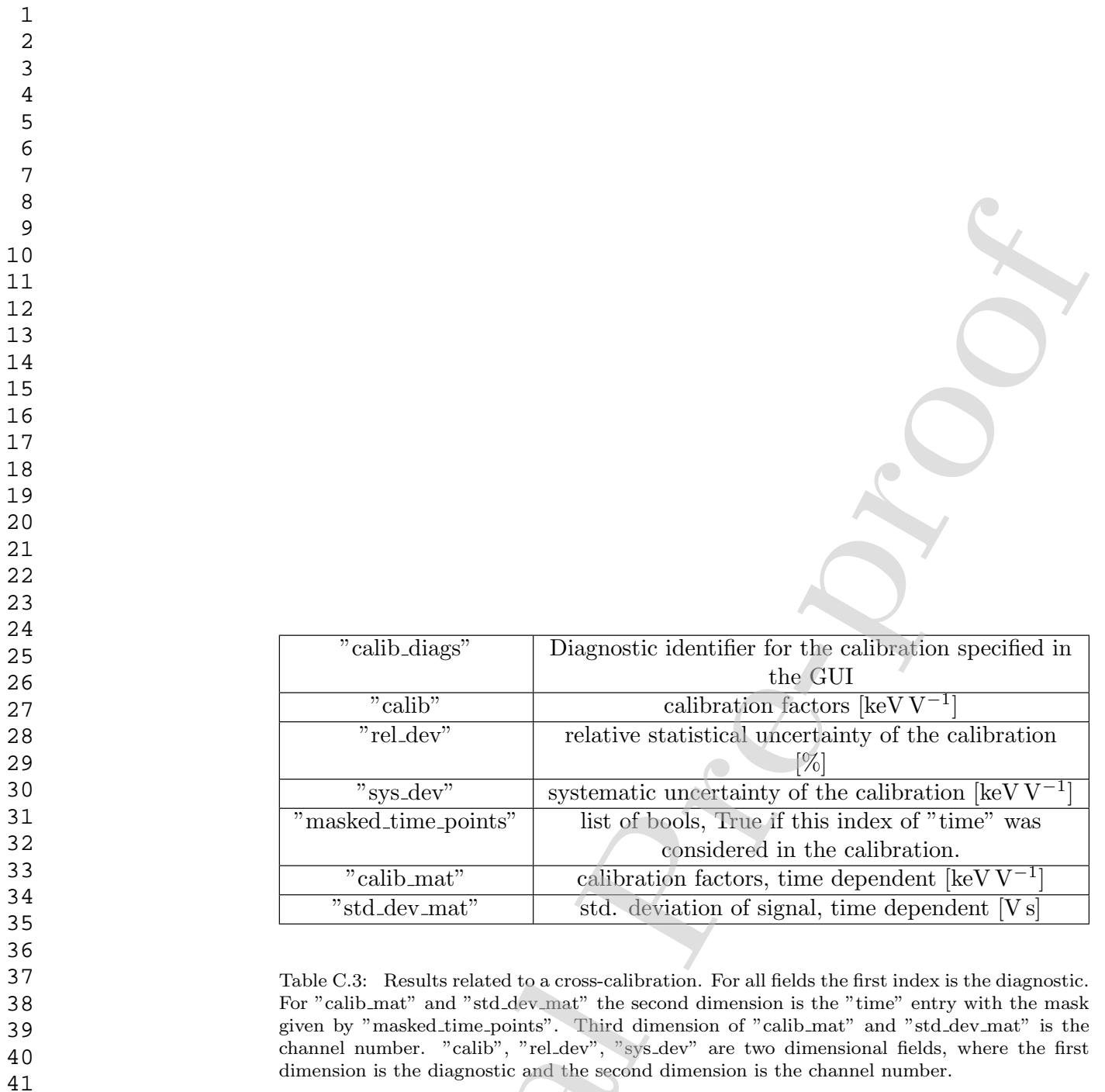




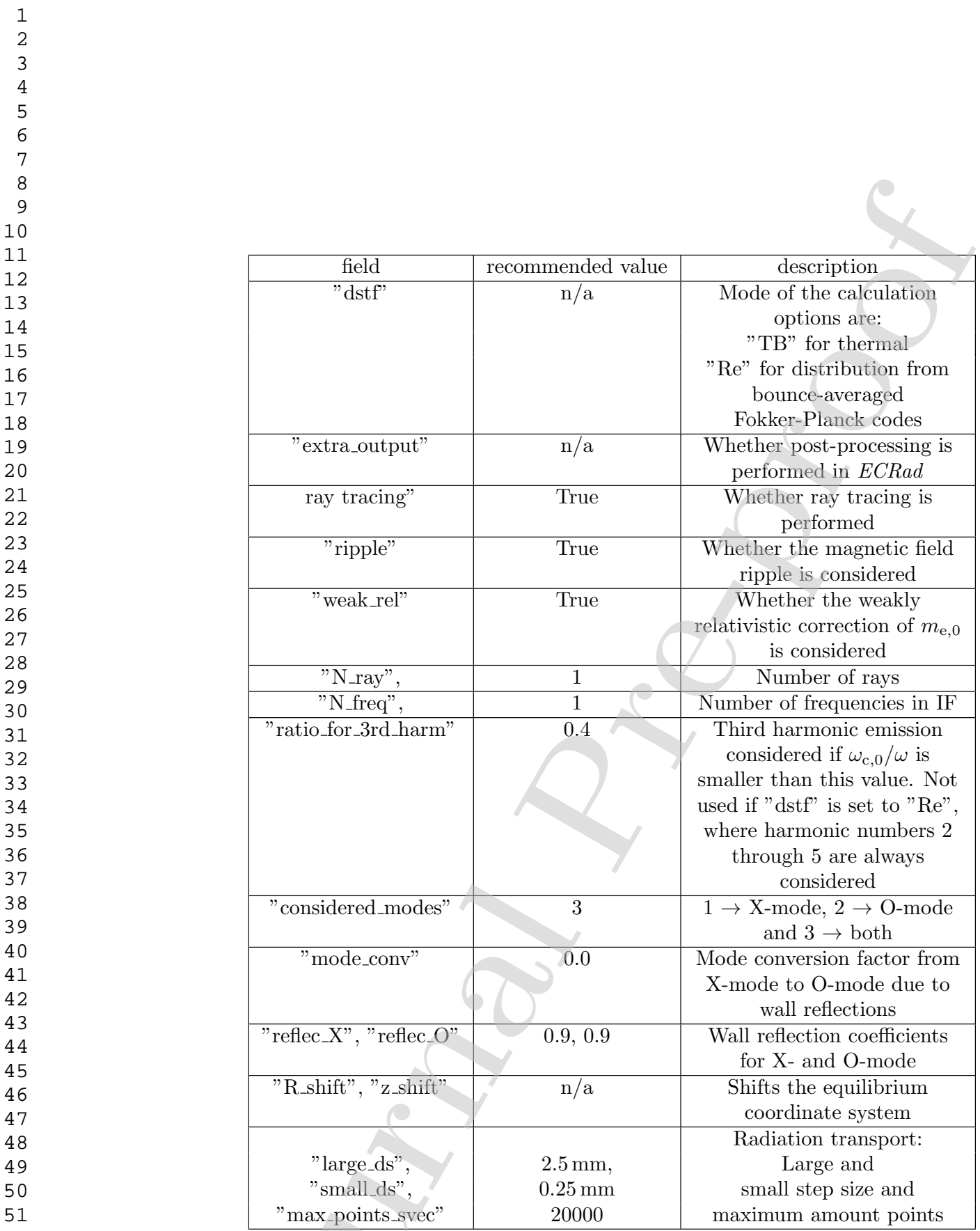

Table C.4: Fields containing the configuration of the ECRad calculation. 
Table C.5: Fields containing the scenario considered in the ECRad calculation. Can be used as input of GUI. Launch geometry follows the convention of the TORBEAM code [70]. All quantities are either in SI-units or dimensionless. All quantities except "vessel_bd" and the scaling parameters have time as first index. All parameters with "launch" have the channel number as second index. 
[1] E. d. 1. Luna, J. Sánchez, V. Tribaldos, J.-E. contributors, G. Conway, W. Suttrop, J. Fessey, R. Prentice, C. Gowers, and J. M. Chareau, Review of Scientific Instruments 75, 3831 (2004), URL http://scitation.aip. org/content/aip/journal/rsi/75/10/10.1063/1.1781376.

[2] S. Schmuck, J. Fessey, T. Gerbaud, B. Alper, M. Beurskens, E. de la Luna, A. Sirinelli, M. Zerbini, and J.-E. Contributors, Review of Scientific Instruments 83, 125101 (2012).

[3] C. Sozzi, A. Bruschi, A. Simonetto, E. DeLaLuna, J. Fessey, V. Riccardo, J.-E. Contributors, et al., Fusion engineering and design 74, 691 (2005).

[4] S. K. Rathgeber, L. Barrera, T. Eich, R. Fischer, B. Nold, W. Suttrop, M. Willensdorfer, E. Wolfrum, and the ASDEX Upgrade Team, Plasma Physics and Controlled Fusion 55, 025004 (2013), URL http://stacks . iop.org/0741-3335/55/i=2/a=025004.

[5] I. G. J. Classen, C. W. Domier, N. C. Luhmann, A. V. Bogomolov, W. Suttrop, J. E. Boom, B. J. Tobias, A. J. H. Donné, and A. U. Team, Review of Scientific Instruments $\mathbf{8 5}$ (2014).

[6] S. J. Freethy, T. Görler, A. J. Creely, G. D. Conway, T. Happel, C. Koenen, P. Hennequin, A. E. White, and A. U. Team, Physics of Plasmas 25, 055903 (2018), https://doi.org/10.1063/1.5018930, URL https://doi.org/ $10.1063 / 1.5018930$

[7] M. E. Austin and J. Lohr, Review of Scientific Instruments 74, 1457 (2003), https://doi.org/10.1063/1.1530387, URL https://doi.org/ $10.1063 / 1.1530387$.

[8] B. Tobias, C. Domier, T. Liang, X. Kong, L. Yu, G. Yun, H. Park, I. J. Classen, J. Boom, A. Donné, et al., Review of Scientific Instruments 81, 10D928 (2010).

[9] A. White, L. Schmitz, W. Peebles, T. Carter, T. Rhodes, E. Doyle, P. Gourdain, J. Hillesheim, G. Wang, C. Holland, et al., Review of Scientific Instruments 79, 103505 (2008).

[10] S. Schmuck, H.-J. Hartfuss, M. Hirsch, and T. Stange, Fusion Engineering and Design 84, 1739 (2009).

[11] Y. Nagayama, K. Kawahata, S. Inagaki, S. Kubo, and K. Narihara, in The 30th International Conference on Plasma Science, 2003. ICOPS 2003. IEEE Conference Record - Abstracts. (2003), pp. 271-, ISSN 0730-9244.

[12] A. Mase, Y. Kogi, M. Ohashi, S. Ohsako, Y. Nagayama, K. Kawahata, S. Aoi, and E. Sakata, Review of scientific instruments 74, 1445 (2003).

[13] Y. Kogi, T. Sakoda, A. Mase, N. Ito, Y. Yokota, S. Yamaguchi, Y. Nagayama, S. H. Jeong, M. Kwon, and K. Kawahata, Review of Scientific Instruments 79, 10F115 (2008). 
[14] G. Yun, W. Lee, M. Choi, J. Kim, H. Park, C. Domier, B. Tobias, T. Liang, X. Kong, N. Luhmann Jr, et al., Review of Scientific Instruments 81, 10D930 (2010).

[15] I. Classen, J. Boom, W. Suttrop, E. Schmid, B. Tobias, C. Domier, N. Luhmann Jr, A. Donné, R. Jaspers, P. de Vries, et al., Review of Scientific Instruments 81, 10D929 (2010).

[16] C. Watts, Fusion Science and Technology 52, 176 (2007)

[17] S. S. Denk et al., in 19th Joint workshop (EC-19) on Electron Cyclotron Emission (ECE) and Electron cyclotron Resonance Heating (ECRH) (EPJ, 2016).

[18] M. Willensdorfer, S. S. Denk, E. Strumberger, W. Suttrop, B. Vanovac, D. Brida, M. Cavedon, I. Classen, M. Dunne, S. Fietz, et al., Plasma Physics and Controlled Fusion 58, 114004 (2016), URL http://stacks . iop. org/0741-3335/58/i=11/a=114004.

[19] P. Blanchard, S. Alberti, S. Coda, H. Weisen, P. Nikkola, and I. Klimanov, Plasma Physics and Controlled Fusion 44, 2231 (2002), URL http://stacks.iop.org/0741-3335/44/i=10/a=310.

[20] S. Coda and T. Team, in Proceedings of the 23rd IAEA Fusion Energy Conference (2010), CONF, pp. OV-5.

[21] J. Mlynar, O. Ficker, E. Macusova, T. Markovic, D. Naydenkova, G. Papp, J. Urban, M. Vlainic, P. Vondracek, V. Weinzettl, et al., Plasma Physics and Controlled Fusion 61, 014010 (2018).

[22] R. W. Harvey, M. R. O'Brien, V. V. Rozhdestvensky, T. C. Luce, M. G. McCoy, and G. D. Kerbel, Physics of Fluids B: Plasma Physics 5, 446 (1993), https://doi.org/10.1063/1.860530, URL https://doi.org/10.1063/ 1.860530 .

[23] G. Bekefi, Radiation processes in plasmas, Wiley series in plasma physics (Wiley, 1966), URL https://books.google.de/books?id= CMI8AAAAIAAJ.

[24] D. Farina, L. Figini, P. Platania, and C. Sozzi, AIP Conference Proceedings 988, 128 (2008), https://aip.scitation.org/doi/pdf/10. 1063/1.2905053, URL https://aip.scitation.org/doi/abs/10.1063/ 1.2905053.

[25] N. Marushchenko, Y. Turkin, and H. Maassberg, Computer Physics Communications 185, 165 (2014), ISSN 0010-4655, URL http://www . sciencedirect.com/science/article/pii/S0010465513003032.

[26] R. Sillen, W. Goedheer, M. Allaart, and A. Kattenberg, Tech. Rep., Associatie Euratom-FOM (1987). 
[27] N. B. Marushchenko, A. Dinklage, H. Hartfuss, M. Hirsch, H. Maaßberg, and Y. Turkin, Fusion science and technology 50, 395 (2006).

[28] T. Zhou, Y. Liu, A. Ti, L. Figini, H. Zhao, Z. Zhu, and B. Ling, Fusion Science and Technology 74, 154 (2018).

[29] S. Schmuck, J. Svensson, E. De La Luna, L. Figini, T. Johnson, B. Alper, M. Beurskens, J. Fessey, T. Gerbaud, A. Sirinelli, et al., in 38th EPS Conference on Plasma Physics (European Physical Society, 2011).

[30] L. Barrera, E. De la Luna, L. Figini, M. Beurskens, M. Brix, F. Castejón, P. de Vries, D. Farina, M. Kempenaars, P. Lomas, et al., Plasma Physics and Controlled Fusion 52, 085010 (2010).

[31] R. Fischer, C. J. Fuchs, B. Kurzan, W. Suttrop, E. Wolfrum, and the ASDEX Upgrade Team, Fusion Science and Technology 58, 675 (2010), ISSN 1536-1055.

[32] S. S. Denk, R. Fischer, H. M. Smith, P. Helander, O. Maj, E. Poli, J. Stober, U. Stroth, W. Suttrop, E. Westerhof, et al., Plasma Physics and Controlled Fusion 60, 105010 (2018), URL http://stacks.iop.org/0741-3335/60/ $i=10 / a=105010$.

[33] B. Vanovac, E. Wolfrum, S. S. Denk, F. Mink, F. M. Laggner, G. Birkenmeier, M. Willensdorfer, E. Viezzer, M. Hoelzl, S. J. Freethy, et al., Plasma Physics and Controlled Fusion 60, 045002 (2018), URL http: //stacks. iop.org/0741-3335/60/i=4/a=045002.

[34] B. Vanovac, E. Wolfrum, M. Hoelzl, M. Willensdorfer, M. Cavedon, G. Harrer, F. Mink, S. Denk, S. Freethy, M. Dunne, et al., Nuclear Fusion 58, 112011 (2018).

[35] B. Vanovac, S. Denk, E. Wolfrum, M. Willensdorfer, W. Suttrop, R. Fischer, and N. Luhmann, in EPJ Web of Conferences (EDP Sciences, 2019), vol. 203, p. 02011.

[36] S. J. Freethy, T. Görler, A. J. Creely, G. D. Conway, S. S. Denk, T. Happel, P. Henniquin, C. Koenen, and A. E. White, in EPJ Web of Conferences (EDP Sciences, 2019), vol. 203, p. 03001.

[37] S. S. Denk, Ph.D. thesis, Technische Universität München (2019).

[38] U. Bellotti, M. Bornatici, and F. Engelmann, La Rivista del Nuovo Cimento (1978-1999) 20, 1 (1997).

[39] D. Farina, Fusion Science and Technology 52, 154 (2007), ISSN 1536-1055, $14^{\text {th }}$ Joint Workshop on Electron Cyclotron Emission and Electron Cyclotron Resonance Heating, Santorini, GREECE, MAY 09-12, 2006. 
[40] M. Bornatici, R. Cano, O. D. Barbieri, and F. Engelmann, Nuclear Fusion 23, 1153 (1983), URL http://stacks.iop.org/0029-5515/23/i=9/ $\mathrm{a}=005$.

[41] E. Poli, A. Peeters, and G. Pereverzev, Computer Physics Communications 136, 90 (2001), ISSN 0010-4655, URL http://www.sciencedirect.com/ science/article/pii/S0010465501001461.

[42] E. Poli, A. Bock, M. Lochbrunner, O. Maj, M. Reich, A. Snicker, A. Stegmeir, F. Volpe, N. Bertelli, R. Bilato, et al., Computer Physics Communications 225, 36 (2018).

[43] A. Kritz, H. Hsuan, R. Goldfinger, and D. Batchelor, in Heating in Toroidal Plasmas 1982 (Elsevier, 1982), pp. 707-723.

[44] E. Westerhof, Tech. Rep., Stichting voor Fundamenteel Onderzoek der Materie (1989).

[45] E. Mazzucato, Physics of Fluids B 4, 3460 (1992).

[46] E. Westerhof, Plasma Physics and Controlled Fusion 39, 1015 (1997), URL http: //stacks . iop.org/0741-3335/39/i=6/a=007.

[47] H. Bindslev, Plasma Physics and Controlled Fusion 35, 1093 (1993), URL http://stacks.iop.org/0741-3335/35/i=9/a=002.

[48] D. Farina, Fusion Science and Technology 53, 130 (2008).

[49] H. Weitzner and D. Batchelor, The Physics of Fluids 23, 1359 (1980).

[50] I. Hutchinson, Principles of Plasma Diagnostics (Cambridge University Press, 1987), ISBN 9780521326223, URL https://books.google.de/ books?id=iSy-QgAACAAJ.

[51] H. Bindslev, in EC-9: Proceedings of the Ninth Joint Workshop on Electron Cyclotron Emission and Electron Cyclotron Heating (World Scientific, 1995).

[52] F. Albajar, N. Bertelli, M. Bornatici, and F. Engelmann, Plasma Physics and Controlled Fusion 49, 15 (2007), ISSN 0741-3335.

[53] T. H. Stix, The Theory of Plasma Waves (McGraw-Hill, 1962).

[54] M. Bornatici and F. Engelmann, Physics of Plasmas 1, 189 (1994), ISSN $1070-664 \mathrm{X}$

[55] G. Garstka, M. Austin, and R. Ellis, Fusion Engineering and Design 53, 123 (2001), ISSN 0920-3796, URL http://www.sciencedirect.com/science/ article/pii/S0920379600004865.

[56] I. G. J. Classen, Ph.D. thesis, Technische Universiteit Eindhoven (2007). 
[57] V. Tribaldos, EFDA-JET Reports 2 (2001).

[58] G. Van Rossum and F. L. Drake Jr, Python reference manual (Centrum voor Wiskunde en Informatica Amsterdam, 1995), URL http://www.cs.cmu.edu/afs/cs.cmu.edu/project/gwydion-1/ OldFiles/OldFiles/python/Doc/ref.ps.

[59] R. Dux, private communication.

[60] P. Dierckx, Curve and surface fitting with splines (Oxford University Press, 1995).

[61] H. Kogelnik and T. Li, Appl. Opt. 5, 1550 (1966), URL http://ao.osa. org/abstract .cfm?URI=ao-5-10-1550.

[62] A. C. Hindmarsh, Scientific Computing 1, 55 (1983).

[63] W. H. M. Clark, Plasma Physics 25, 1501 (1983), URL http://stacks . iop. org $/ 0032-1028 / 25 / i=12 / a=315$.

[64] R. Prater, Physics of Plasmas 11, 2349 (2004), https://doi.org/10. 1063/1.1690762, URL https://doi.org/10.1063/1.1690762.

[65] N. Rappin and R. Dunn, wxPython in Action (Manning Publications Co., Greenwich, CT, USA, 2006), ISBN 1932394621.

[66] H. J. Hartfuss, T. Geist, and M. Hirsch, Plasma Physics and Controlled Fusion 39, 1693 (1997), URL http://stacks.iop.org/0741-3335/39/i= $11 / \mathrm{a}=001$.

[67] J. Rasmussen, S. Nielsen, M. Stejner, M. Salewski, A. S. Jacobsen, S. B. Korsholm, F. Leipold, F. Meo, P. K. Michelsen, D. Moseev, et al., in American Institute of Physics Conference Series (2014), vol. 1612 of American Institute of Physics Conference Series, pp. 117-120, 1310.2406.

[68] M. Willensdorfer, E. Strumberger, W. Suttrop, R. Dunne, M aner, G. Birkenmeier, D. Brida, M. Cavedon, S. Denk, V. Igochine, et al., Nuclear Fusion 57, 116047 (2017).

[69] U. G. Matlab, Inc., Natick, MA 1992 (1760).

[70] Computer Physics Communications 225, 36 (2018), ISSN 00104655, URL http://www.sciencedirect.com/science/article/pii/ S001046551730423X. 
Declaration of Interest Statement

\section{Declaration of interests}

$\bigotimes$ The authors declare that they have no known competing financial interests or personal relationships that could have appeared to influence the work reported in this paper.

$\square$ The authors declare the following financial interests/personal relationships which may be considered as potential competing interests: 